\title{
ANCHORED VECTOR BUNDLES AND LIE ALGEBROIDS
}

\author{
PAUL POPESCU and MARCELA POPESCU \\ P.O. Box 4-66, Craiova 1100, Romania \\ E-mail:paulpopescu@email.com, marcelapopescu@email.com
}

\begin{abstract}
The derived generalized algebroid and the derived generalized Lie algebroid of an anchored vector bundle are defined. Some natural functors from the two categories of anchored vector bundles to the corresponding categories of generalized algebroids and generalized Lie algebroids respectively are also considered.

A natural result is proved: the derived (Lie) algebroid of an anchored vector subbundle is a generalized (Lie) algebroid of the underlying bundle. Lifts of linear R-connections and skewsymmetric forms respectively are constructed and the modular class of an almost Lie structure is defined.
\end{abstract}

1. Introduction. The algebraic approach of the category of Lie algebroids [7] and its abstract version, the Lie pseudoalgebras [10], can be enlarged to the categories of vector bundles with differentials and their abstract versions, the modules with differentials [14].

An anchored vector bundle denoted as AVB (or a relative tangent space in $[12,13,14]$ ) is a vector bundle $\theta=(R, t, M)$ together a vector bundle morphism $D: \theta \rightarrow \tau M$, called an anchor. A bracket (or a Lie map) on an $\operatorname{AVB}(\theta, D)$ is a map $[\cdot, \cdot]_{\theta}: \Gamma(\theta) \times \Gamma(\theta) \rightarrow \Gamma(\theta)$ which enjoys the properties that it is bilinear over $\mathbb{R}$, is skew symmetric and $[X, f Y]_{\theta}=$ $(D X)(f) Y+f[X, Y]_{\theta},(\forall) X, Y \in \Gamma(\theta)$ and $f \in \mathcal{F}(M)$. An almost Lie structure (ALS) is a triple $\left(\theta, D,[\cdot, \cdot]_{\theta}\right)$. An algebroid is an $\operatorname{ALS}\left(\theta, D,[\cdot, \cdot]_{\theta}\right)$ which enjoys the property that $[D X, D Y]=D[X, Y]_{\theta},(\forall) X, Y \in \Gamma(\theta)$, where the first bracket is the Lie bracket on $\mathcal{X}(M)$. A Lie algebroid is an algebroid $\left(\theta, D,[\cdot, \cdot]_{\theta}\right)$ which has a null Jacobiator, i.e. $\mathcal{J}(X, Y, Z) \equiv \sum_{\text {cycl. }}\left[[X, Y]_{\theta}, Z\right]_{\theta}=0,(\forall) X, Y, Z \in \Gamma(\theta)$. The anchored vector bundles, the almost Lie structures, the algebroids and the Lie algebroids are called in [14] vector bundles with differentials. A differential calculus using an ALS is constructed in [13]. In this paper one consider vector bundles and admissible vector bundles, i.e. vector bundles on a finite dimensional manifold, but which may have an infinite dimensional fiber. Notice that the morphisms of vector bundles and vector bundles with differentials come from the morphisms of the contravariant category of modules (see [14] for more details).

2000 Mathematics Subject Classification: 18F15, 55R25, 22A30, 18B40, 58H99.

The paper is in final form and no version of it will be published elsewhere. 
To every anchored vector bundle $(\theta, D)$ one can associate a generalized (i.e. with an infinite dimensional fiber) algebroid $\left(\theta^{(\infty)}, D^{(\infty)},[\cdot, \cdot]_{\theta(\infty)}\right)$ and a generalized Lie algebroid $\left(\theta^{\infty}, D^{\infty},[\cdot, \cdot]_{\theta^{\infty}}\right)$, which belongs to an isomorphism class of generalized algebroids and generalized Lie algebroids respectively, which does not depend on the linear R-connection or the bracket, used as ingredients in the construction. We call them the derived generalized algebroid and the derived generalized Lie algebroid respectively of the anchored vector bundle $(\theta, D)$. This correspondence related to the anchored vector bundles can be enriched to morphisms, defining in this way four covariant functors (called the derived functors) from the two categories of anchored vector bundles to the two categories of generalized algebroids and the two categories of generalized Lie algebroids respectively. In the same way as a geometric distribution is defined (using the Lie algebroid of the tangent bundle) the derived generalized algebroid and the derived generalized Lie algebroid contains the given anchored vector bundle and induces an almost Lie structure on the bundle. This construction can be interpreted as well in therms of non-holonomic spaces of G. Vrănceanu [24], since a linear R-connection and its curvature, which is no more a tensor, but a new linear R-connection on a suitable anchored vector bundle, are used as essential ingredients. If $\left(\theta^{\prime}, D^{\prime}\right)$ is an anchored vector subbundle of $(\theta, D)$, then $\left(\theta^{\prime(\infty)}, D^{\prime(\infty)},[\cdot, \cdot]_{\theta^{\prime}(\infty)}\right)$ and $\left(\theta^{\prime \infty}, D^{\prime \infty},[\cdot, \cdot]_{\theta^{\prime} \infty}\right)$ are generalized (Lie) subalgebroids of $\left(\theta^{(\infty)}, D^{(\infty)},[\cdot, \cdot]_{\theta}(\infty)\right)$ and of $\left(\theta^{\infty}, D^{\infty},[\cdot, \cdot]_{\theta}\right)$ respectively. Particularly, if $\mathcal{D} \subset \tau M$ is a regular distribution, then $\mathcal{D}^{(\infty)}$ is a generalized sub-algebroid of $\tau M^{(\infty)}$ and $\mathcal{D}^{\infty}$ is a generalized Lie subalgebroid of $\tau M^{\infty}$.

Let $(\theta, D)$ be an AVB, $\theta^{\prime}$ be a vector bundle, over the same base as $\theta$, and $\nabla$ : $\Gamma(\theta) \times \Gamma\left(\theta^{\prime}\right) \rightarrow \Gamma\left(\theta^{\prime}\right)$ be a linear R-connection on $\theta^{\prime}$. Then there are linear R-connections $\nabla^{(\infty)}: \Gamma\left(\theta^{(\infty)}\right) \times \Gamma\left(\theta^{\prime}\right) \rightarrow \Gamma\left(\theta^{\prime}\right)$ and $\nabla^{\infty}: \Gamma\left(\theta^{\infty}\right) \times \Gamma\left(\theta^{\prime}\right) \rightarrow \Gamma\left(\theta^{\prime}\right)$ which are curvature free and extend $\nabla$. For $p \geq 1$ any $p$-form $\omega \in \Gamma\left(\wedge^{p} \theta^{*}\right)$ can be lifted canonically to a closed $p$-form $\omega_{(\infty)} \in \Gamma\left(\wedge^{p}\left(\theta^{*}\right)^{(\infty)}\right)$. A 1-form $\omega \in \Gamma\left(\theta^{*}\right)$ can be lifted canonically to a closed 1 -form $\omega_{\infty} \in \Gamma\left(\theta^{(\infty) *}\right)$. These construction enable us to define the modular class of an almost Lie structure.

Notice that the essential constructions made above on anchored vector bundles have a pure algebraic feature, since they can be performed also for modules with differentials.

All the manifolds and vector bundles are real and smooth (i.e. of class $C^{\infty}$ ).

2. The derived algebroid of an anchored vector bundle. Let us consider a given anchored vector bundle $(\theta, D)$, where $\theta=(R, \pi, M)$. A linear relative connection (or a linear $R$-connection in brief) related to $(\theta, D)$ on a vector bundle $\xi=(E, p, M)$, is a map $\nabla: \Gamma(\theta) \times \Gamma(\xi) \rightarrow \Gamma(\xi), \nabla(X, u) \stackrel{\text { not. }}{=} \nabla_{X} u$, such that the Koszul conditions:

$$
\begin{gathered}
\nabla_{X+Y} u=\nabla_{X} u+\nabla_{Y} u, \nabla_{f X} u=f \nabla_{X} u \\
\nabla_{X}(u+v)=\nabla_{X} u+\nabla_{X} v, \nabla_{X}(f u)=D(X)(f) u+f \nabla_{X} u,
\end{gathered}
$$

$(\forall) X, Y \in \Gamma(\theta)$ and $u \in \Gamma(\xi)$, hold. If $\left(\theta, D,[\cdot, \cdot]_{\theta}\right)$ is an ALS, the curvature of $\nabla$ is $\nabla_{X \wedge Y}=\nabla_{X} \nabla_{Y}-\nabla_{Y} \nabla_{X}-\nabla_{[X, Y]_{\theta}}$ Particularly, a linear R-connection on an ALS $(\theta, D)$ can be defined. Its torsion is $T(X, Y)=\nabla_{X} Y-\nabla_{Y} X-[X, Y]_{\theta}$. The formula $[X, Y]_{\theta}^{\prime}=\nabla_{X} Y-\nabla_{Y} X$ defines a bracket, thus $(\theta, D)$ becomes an ALS $\left(\theta, D,[\cdot, \cdot]_{\theta}^{\prime}\right)$ with 
respect to which $\nabla$ is torsion free. Conversely, given a linear R-connection $\nabla$ and an ALS $\left(\theta, D,[\cdot, \cdot]_{\theta}\right)$, then the new linear R-connection $\nabla^{\prime}$ given by $\nabla_{X}^{\prime} Y=\nabla_{X} Y-\frac{1}{2} T(X, Y)$ is torsion free. Using a new bracket given by $[X, Y]_{\theta}^{\prime}=[X, Y]_{\theta}+T(X, Y)$, the linear R-connection $\nabla$ is torsion free.

Using the same idea of proof of the theorem of existence of usual linear connections on vector bundles (i.e. when the anchor is the identity on the tangent bundle), it can be proved that giving an anchored vector bundle $(\theta, D)$, then every vector bundle over the same base as $\theta$ allows a linear R-connection. Taking into account the above remarks, an anchored vector bundle allows always a torsion free linear R-connection.

If $\left(\theta, D,[\cdot, \cdot]_{\theta}\right)$ is an almost Lie structure, then a vector bundle morphism $\mathcal{D}: \theta \wedge \theta \rightarrow$ $\tau M$ is induced by the module morphism of sections:

$$
\begin{aligned}
& \mathcal{D}: \Gamma(\theta \wedge \theta) \cong \Gamma(\theta) \wedge_{\mathcal{F}(M)} \Gamma(\theta) \rightarrow \mathcal{X}(M), \\
& \mathcal{D}(X \wedge Y)=[D(X), D(Y)]-D\left([X, Y]_{\theta}\right) .
\end{aligned}
$$

Thus $\mathcal{D}$ is an anchor map for $\theta \wedge \theta$, i.e. $(\theta \wedge \theta, \mathcal{D})$ is an anchored vector bundle.

Notice that the curvature of a linear R-connection $\nabla$ on $\theta$ is a linear R-connection on the anchored vector bundle $(\theta \wedge \theta, \mathcal{D})$ defined above. The formula

$$
[X \wedge Y, U \wedge V]_{\theta \wedge \theta}=\nabla_{X \wedge Y}(U \wedge V)-\nabla_{U \wedge V}(X \wedge Y)
$$

defines a bracket on $(\theta \wedge \theta, \mathcal{D})$, thus $\left(\theta \wedge \theta, \mathcal{D},[\cdot, \cdot]_{\theta \wedge \theta}\right)$ becomes an ALS.

Consider now on the vector bundle $\theta^{(1)}=\theta \oplus(\theta \wedge \theta)$ the anchor given by $D^{(1)}(X+$ $(Y \wedge Z))=D(X)+\mathcal{D}(Y \wedge Z)$ and the bracket given by the formulas

$$
\begin{aligned}
{[X, Y]_{\theta^{(1)}} } & =[X, Y]_{\theta}+X \wedge Y, \\
{[X \wedge Y, Z]_{\theta^{(1)}} } & =\nabla_{X \wedge Y} Z-\nabla_{Z}(X \wedge Y), \\
{[X \wedge Y, Z \wedge T]_{\theta^{(1)}} } & =\nabla_{X \wedge Y}(Z \wedge T)-\nabla_{Z \wedge T}(X \wedge Y) .
\end{aligned}
$$

We call the almost Lie structure $\left(\theta^{(1)}, D^{(1)},[\cdot, \cdot]_{\theta^{(1)}}\right)$ the (first) derived ALS of $\left(\theta, D,[\cdot, \cdot]_{\theta}\right)$, given by $\nabla$.

Proposition 2.1. The following properties hold true:

1. $\left[D^{(1)}(X), D^{(1)}(Y)\right]=D^{(1)}\left([X, Y]_{\theta^{(1)}}\right),(\forall) X, Y \in \Gamma(\theta)$.

2. If the linear $R$-connection $\nabla$ has no torsion, then $\mathcal{J}^{(1)}(X, Y, Z)=0,(\forall) X, Y, Z \in$ $\Gamma(\theta)$, where $\mathcal{J}^{(1)}$ denotes the Jacobiator of $[\cdot, \cdot]_{\theta^{(1)}}$.

Proof. We have: $\left[D^{(1)}(X), D^{(1)}(Y)\right]=[D(X), D(Y)]=D\left([X, Y]_{\theta}\right)+\mathcal{D}(X \wedge Y)=$ $D\left([X, Y]_{\theta}\right)+D^{(1)}(X \wedge Y)=D^{(1)}\left([X, Y]_{\theta}+X \wedge Y\right)=D^{(1)}\left([X, Y]_{\theta^{(1)}}\right)$.

For the second equality we have: $\left[[X, Y]_{\theta^{(1)}}, Z\right]_{\theta^{(1)}}=\left[[X, Y]_{\theta}+X \wedge Y, Z\right]_{\theta^{(1)}}=$ $\left[[X, Y]_{\theta}, Z\right]_{\theta}+[X, Y]_{\theta} \wedge Z+\nabla_{X \wedge Y} Z-\nabla_{Z}(X \wedge Y)=\left[[X, Y]_{\theta}, Z\right]_{\theta}+[X, Y]_{\theta} \wedge Z+$ $\nabla_{X} \nabla_{Y} Z-\nabla_{Y} \nabla_{X} Z-\nabla_{[X, Y]_{\theta}} Z-\nabla_{Z} X \wedge Y-X \wedge \nabla_{Z} Y=-\nabla_{Z}[X, Y]_{\theta}+\nabla_{X} \nabla_{Y} Z-$ $\nabla_{Y} \nabla_{X} Z-\nabla_{X} Y \wedge Z-\nabla_{Y} X \wedge Z+\nabla_{Z} X \wedge Y-X \wedge \nabla_{Z} Y$. So, [[X,Y] $\left.]_{\theta^{(1)}}, Z\right]_{\theta^{(1)}}=$ $-\nabla_{Z} \nabla_{X} Y+\nabla_{Z} \nabla_{Y} X+\nabla_{X} \nabla_{Y} Z-\nabla_{Y} \nabla_{X} Z-\nabla_{X} Y \wedge Z-\nabla_{Y} X \wedge Z+\nabla_{Z} X \wedge Y-X \wedge \nabla_{Z} Y$.

Writing the analogous expressions for $\left.[Y, Z]_{\theta^{(1)}}, X\right]_{\theta^{(1)}}$ and $\left[[Z, X]_{\theta^{(1)}}, Y\right]_{\theta^{(1)}}$, then summing, we obtain the second equality. 
The linear R-connection $\nabla$ on $\theta$ lifts to a torsion free linear R-connection $\nabla^{(1)}$ on $\theta^{(1)}$, according to the formulas:

$$
\begin{aligned}
\nabla_{X}^{(1)} Y & =\nabla_{X} Y+\frac{1}{2} X \wedge Y, \nabla_{X}^{(1)}(Y \wedge Z)=\nabla_{X}(Y \wedge Z), \\
\nabla_{X \wedge Y}^{(1)} Z & =\nabla_{X \wedge Y} Z, \nabla_{X \wedge Y}^{(1)}(Z \wedge T)=\nabla_{X \wedge Y}(Z \wedge T) .
\end{aligned}
$$

Now we define $\left(\theta^{(2)}, D^{(2)}, M\right)$ the second derived $A L S$ of $\left(\theta, D,[\cdot, \cdot]_{\theta}\right)$, as follows:

$$
\theta^{(2)}=\theta \oplus\left(\theta \wedge_{1} \theta\right) \oplus\left(\theta \wedge_{2}\left(\theta \wedge_{1} \theta\right)\right) \oplus\left(\left(\theta \wedge_{1} \theta\right) \wedge_{2}\left(\theta \wedge_{1} \theta\right)\right) .
$$

The anchor $D^{(2)}: \theta^{(2)} \rightarrow \tau M$ is defined by:

$D^{(2)}(X)=D^{(1)}(X),(\forall) X \in \Gamma\left(\theta^{(1)}\right) \cong \Gamma(\theta) \oplus\left(\Gamma(\theta) \wedge_{1} \Gamma(\theta)\right)$ and

$D^{(2)}\left(X \wedge_{2} Y\right)=\left[D^{(1)}(X), D^{(1)}(Y)\right]-D^{(1)}\left([X, Y]_{\theta^{(1)}}\right)$, whenever $\left(X, Y \in \Gamma\left(\theta \wedge_{1} \theta\right) \cong\right.$ $\left.\Gamma(\theta) \wedge_{1} \Gamma(\theta)\right)$ or $\left(X \in \Gamma(\theta)\right.$ and $\left.Y \in \Gamma(\theta) \wedge_{1} \Gamma(\theta)\right)$.

The linear connection $\nabla^{(2)}$ on $\theta^{(2)}$ is defined by:

$\nabla_{X}^{(2)} Y=\nabla_{X} Y+\frac{1}{2} X \wedge_{1} Y=\nabla_{X}^{(1)} Y$, whenever $X, Y \in \Gamma(\theta)$;

$\nabla_{X}^{(2)} Y=\nabla_{X}^{(1)} Y+\frac{1}{2} X \wedge_{2} Y$, whenever $\left(X \in \Gamma(\theta)\right.$ and $\left.Y \in \Gamma(\theta) \wedge_{1} \Gamma(\theta)\right)$ or $(X \in$

$\Gamma(\theta) \wedge_{1} \Gamma(\theta)$ and $\left.Y \in \Gamma(\theta)\right)$ or $\left(X, Y \in \Gamma(\theta) \wedge_{1} \Gamma(\theta)\right)$;

$\nabla_{X \wedge_{2} Y}^{(2)} Z=\nabla_{X}^{(1)} \nabla_{Y}^{(1)} Z-\nabla_{Y}^{(1)} \nabla_{X}^{(1)} Z-\nabla_{[X, Y]_{\theta}(1)}^{(1)} Z,(\forall) X, Y, Z \in \Gamma\left(\theta^{(1)}\right)$;

$\nabla_{X}^{(2)}\left(Y \wedge_{2} Z\right)=\nabla_{X}^{(1)} Y \wedge_{2} Z+Y \wedge_{2} \nabla_{X}^{(1)} Z,(\forall) X, Y, Z \in \Gamma\left(\theta^{(1)}\right)$;

$\nabla_{X \wedge_{2} Y}^{(2)}\left(Z \wedge_{2} T\right)=\nabla_{X \wedge_{2} Y}^{(2)} Z \wedge_{2} T+Z \wedge_{2} \nabla_{X \wedge_{2} Y}^{(1)} T,(\forall) X, Y, Z, T \in \Gamma(\theta) \wedge_{1} \Gamma(\theta)$.

The bracket $[\cdot, \cdot]_{\theta^{(2)}}$ is defined by:

$[X, Y]_{\theta^{(2)}}=[X, Y]_{\theta}+X \wedge_{1} Y=[X, Y]_{\theta^{(1)}},(\forall) X, Y \in \Gamma(\theta)$

$[X, Y]_{\theta^{(2)}}=[X, Y]_{\theta^{(1)}}+X \wedge_{2} Y,(\forall) X \in \Gamma(\theta)$ and $Y \in \Gamma(\theta) \wedge_{1} \Gamma(\theta)$, or $X, Y \in \Gamma(\theta) \wedge_{1} \Gamma(\theta)$; $\left[X \wedge_{2} Y, Z\right]_{\theta^{(2)}}=\nabla_{X \wedge_{2} Y}^{(2)} Z-\nabla_{Z}^{(2)} X \wedge_{2} Y,(\forall) X, Y, Z \in \Gamma\left(\theta^{(1)}\right)$;

$\left[X \wedge_{2} Y, Z \wedge_{2} T\right]_{\theta^{(2)}}=\nabla_{X \wedge_{2} Y}^{(2)}\left(Z \wedge_{2} T\right)-\nabla_{Z \wedge_{2} T}^{(2)}\left(X \wedge_{2} Y\right),(\forall) X, Y, Z, T \in \Gamma(\theta) \wedge_{1} \Gamma(\theta)$.

It is easy to see that the R-linear connection $\nabla^{(2)}$ on $\theta^{(2)}$ is torsion free.

The derived almost Lie structure of order $p \in \mathbb{N}$ (or the $p$-derived $A L S$ ), denoted as $\left(\theta^{(p)}, D^{(p)}, M\right)$, is obtained inductively for $p \geq 2$ as follows. Assuming $\theta^{(k)}$ constructed for $0 \leq k \leq p-1$ and0 $\theta^{(p-1)}=\theta^{(p-2)} \oplus \bar{\theta}^{(p)}$, then $\theta^{(p)}=\theta^{(p-1)} \oplus\left(\theta^{(p-1)} \wedge_{p} \bar{\theta}^{(p)}\right)$. The anchor map $D^{(p)}$ on $\theta^{(p)}$ is defined by:

$D^{(p)}(X)=D^{(p-1)}(X)$, whenever $X \in \Gamma\left(\theta^{(p-1)}\right)$;

$D^{(p)}\left(X \wedge_{p} Y\right)=\left[D^{(p-1)}(X), D^{(p-1)}(Y)\right]-D^{(p-1)}\left([X, Y]_{\theta^{(p-1)}}\right)$, whenever $X \in \Gamma\left(\theta^{(p-1)}\right)$ and $Y \in \Gamma\left(\bar{\theta}^{(p)}\right)$.

The linear connection $\nabla^{(p)}$ on $\theta^{(p)}$ is defined by:

$\nabla_{X}^{(p)} Y=\nabla_{X}^{(p-2)} Y+\frac{1}{2} X \wedge_{p-1} Y=\nabla_{X}^{(p-1)} Y$, whenever $X, Y \in \Gamma\left(\theta^{(p-2)}\right)$;

$\nabla_{X}^{(p)} Y=\nabla_{X}^{(p-1)} Y+\frac{1}{2} X \wedge_{p} Y$, whenever $\left(X \in \Gamma\left(\theta^{(p-2)}\right)\right.$ and $\left.Y \in \Gamma\left(\bar{\theta}^{(p)}\right)\right)$ or $\left(X \in \Gamma\left(\bar{\theta}^{(p)}\right)\right.$ and $\left.Y \in \Gamma\left(\theta^{(p-2)}\right)\right)$ or $\left(X, Y \in \Gamma\left(\bar{\theta}^{(p)}\right)\right)$; 
$\nabla_{X \wedge_{p} Y}^{(p)} Z=\nabla_{X}^{(p-1)} \nabla_{Y}^{(p-1)} Z-\nabla_{Y}^{(p-1)} \nabla_{X}^{(p-1)} Z-\nabla_{[X, Y]_{\theta}(p-1)}^{(p-1)} Z$, whenever $X, Y, Z \in$ $\Gamma\left(\theta^{(p-1)}\right)$;

$\nabla_{X}^{(p)}\left(Y \wedge_{p} Z\right)=\nabla_{X}^{(p-1)} Y \wedge_{p} Z+Y \wedge_{p} \nabla_{X}^{(p-1)} Z$, whenever $X, Y, Z \in \Gamma\left(\theta^{(p-1)}\right)$;

$\left.\nabla_{X \wedge_{p} Y}^{(p)}\left(Z \wedge_{p} T\right)\right)=\nabla_{X \wedge_{p} Y}^{(p)} Z \wedge_{p} T+Z \wedge_{p} \nabla_{X \wedge_{p} Y}^{(p)} T$, whenever $X, Y, Z, T \in \Gamma\left(\bar{\theta}^{(p)}\right)$.

The bracket on $\theta^{(p)}$ is defined by:

$[X, Y]_{\theta^{(p)}}=[X, Y]_{\theta^{(p-2)}}+X \wedge_{p-1} Y=[X, Y]_{\theta^{(p-1)}}$, whenever $X, Y \in \Gamma\left(\theta^{(p-1)}\right)$, $[X, Y]_{\theta^{(p)}}=[X, Y]_{\theta^{(p-1)}}+X \wedge_{p} Y$, whenever $\left(X \in \Gamma\left(\theta^{(p-2)}\right)\right.$ and $\left.Y \in \Gamma\left(\bar{\theta}^{(p)}\right)\right)$, or $\left(X, Y \in \Gamma\left(\bar{\theta}^{(p)}\right)\right)$

$\left[X \wedge_{p} Y, Z\right]_{\theta^{(p)}}=\nabla_{X \wedge_{p} Y}^{(p)} Z-\nabla_{Z}^{(p)}\left(X \wedge_{p} Y\right)$, whenever $X, Y, Z \in \Gamma\left(\theta^{(p-1)}\right)$;

$\left[X \wedge_{p} Y, Z \wedge_{p} T\right]_{\theta^{(p)}}=\nabla_{X \wedge_{p} Y}^{(p)}\left(Z \wedge_{p} T\right)-\nabla_{Z \wedge_{p} T}^{(p)}\left(X \wedge_{p} Y\right)$, whenever $X, Y, Z, T \in$ $\Gamma\left(\bar{\theta}^{(p)}\right)$.

It is easy to see that the linear R-connection $\nabla^{(p)}$ is torsion free.

The vector bundles $\theta^{(p)}$ (which have finite dimensional fibers) define the vector bundle $\theta^{(\infty)}=\bigcup_{p \geq 0} \theta^{(p)}$ (since $\theta^{(p)} \subset \theta^{(p+1)}$ is a subbundle), which has infinite dimensional fibers.

The degree of $X_{(\infty)} \in \Gamma\left(\theta^{(\infty)}\right)$, denoted as $\operatorname{deg} X_{(\infty)}$, is the smallest $n \in \mathbb{N}$ such that $X_{(\infty)} \in \theta^{(n)}$. The anchor of $X_{(\infty)}$ is $D^{(\infty)}\left(X_{(\infty)}\right)=\sum_{i=0}^{n} D^{(i)}\left(X_{i}\right)$, where $n=\operatorname{deg} X_{(\infty)}$. Notice that if $n=\operatorname{deg} X_{(\infty)}$ and $m=\operatorname{deg} Y_{(\infty)}$ then

$$
\left[X_{(\infty)}, Y_{(\infty)}\right]_{\theta^{(\infty)}}=\sum_{p \leq n, q \leq m}\left[X_{p}, Y_{q}\right]_{\theta^{(\infty)}} .
$$

Definition 2.1. A generalized algebroid is a vector bundle over $M$ which satisfies the following conditions:

1. It is an inductive limit of vector bundles over $M$ having finite dimensional fibers.

2. It allows an anchor and a bracket.

3. Its sections fulfill the conditions of an algebroid.

A generalized Lie algebroid is a generalized algebroid whose sections fulfill the conditions of a Lie algebroid.

A vector bundle which satisfies the condition 1 . will be called an admissible vector bundle.

A morphism of admissible vector bundles is a vector bundle morphism which is induced inductively by morphisms of the underlying vector subbundles with finite dimensional fibers, which define it.

TheOREM 2.1. The triple $\left(\theta^{(\infty)}, D^{(\infty)},[\cdot, \cdot]_{\theta^{(\infty)}}\right)$ is a generalized algebroid.

Proof. We have to prove that $(\forall) X_{(\infty)}, Y_{(\infty)}, Z_{(\infty)} \in \Gamma\left(\theta^{(\infty)}\right)$,

$$
D^{(\infty)}\left(\left[X_{(\infty)}, Y_{(\infty)}\right]_{\theta(\infty)}\right)=\left[D^{(\infty)}\left(X_{(\infty)}\right), D^{(\infty)}\left(Y_{(\infty)}\right)\right]
$$

In order to do this it suffices to prove that the property holds for $X_{p} \in \Gamma\left(\theta^{(p)}\right), X_{p} \in$ $\Gamma\left(\theta^{(p)}\right)$, but it is a simple consequence of 1 ) from Proposition 2.1. 
Notice that if $\left(\theta, D,[\cdot, \cdot]_{\theta}\right)$ is a (Lie) algebroid, then it is not isomorphic to $\left(\theta^{(\infty)}, D^{(\infty)}\right.$, $\left.[\cdot, \cdot]_{\theta(\infty)}\right)$.

Consider the morphism $\Phi: \theta^{(\infty)} \rightarrow \theta$ of admissible vector bundles which associates the $\theta$-component with every element in $\theta^{(\infty)}$. It is clear that for every $X, Y \in \Gamma(\theta)$ we have $[X, Y]_{\theta}=\Phi\left([X, Y]_{\theta(\infty)}\right)$, i.e. the bracket on $\theta$ is induced by the bracket on $\theta^{(\infty)}$. However $\Phi$ is not a morphism of anchored vector bundles.

3. Morphisms of vector bundles with differentials. Consider now an ALS $\left(\theta^{\prime}, D^{\prime},[\cdot, \cdot]_{\theta^{\prime}}\right)$, an algebroid $\left(\theta, D,[\cdot, \cdot]_{\theta}\right)$, where $\theta^{\prime}$ and $\theta$ have the same base $M$ and an anchored vector bundle morphism $f: \theta^{\prime} \rightarrow \theta$ (i.e. $D^{\prime}=D \circ f$, where $D^{\prime}$ and $D$ are the anchors on $\theta^{\prime}$ and $\theta$ respectively). Denote $f=f_{0}$ and define $f_{1}: \theta^{\prime(1)} \rightarrow \theta$, $f_{1 \mid \theta^{\prime}}=f_{0}$ and $f_{1}(X \wedge Y)=\left[f_{0}(X), f_{0}(Y)\right]_{\theta}-f_{0}\left([X, Y]_{\theta^{\prime}}\right),(\forall) X, Y \in \Gamma\left(\theta^{\prime}\right)$. Thus $D^{(1)} \circ f_{1}(X \wedge Y)=D^{(1)}\left(\left[f_{0}(X), f_{0}(Y)\right]_{\theta}\right)-D^{(1)} \circ f_{0}\left([X, Y]_{\theta^{\prime}}\right)=\left[D^{(1)} \circ f_{0}(X), D^{(1)} \circ\right.$ $\left.f_{0}(Y)\right]-D^{(1)} \circ f_{0}\left([X, Y]_{\theta^{\prime}}\right)=\left[D^{\prime(1)}(X), D^{\prime(1)}(Y)\right]-D^{\prime(1)}\left([X, Y]_{\theta^{\prime}}\right)=D^{\prime(1)}(X \wedge Y)$. It follows that $f_{1}$ is an anchored vector bundle morphism.

Inductively, assume that $f_{k}$ is constructed, then let $f_{k+1}: \theta^{\prime(k+1)} \rightarrow \theta, f_{k+1 \mid \theta^{\prime \prime}(k)}=f_{k}$ and $f_{k+1}\left(X \wedge_{k} Y\right)=\left[f_{k}(X), f_{k}(Y)\right]_{\theta}-f_{k}\left([X, Y]_{\theta^{\prime}(k)}\right),(\forall) X, Y \in \Gamma\left(\theta^{\prime(k)}\right)$. As in the case $k=0$, it can be shown that $f_{k+1}$ is an anchored vector bundle morphism. The maps $\left(f_{k}\right)_{k \in \mathbb{N}}$ define a map $f_{(\infty)}: \theta^{\prime(\infty)} \rightarrow \theta$.

A morphism of generalized (Lie) algebroids over the same base is a morphism of admissible vector bundles $f: \theta^{\prime} \rightarrow \theta$ such that $D^{\prime}=D \circ f$, where $D^{\prime}$ and $D$ are the anchors on $\theta^{\prime}$ and $\theta$ respectively, and $[f(X), f(Y)]_{\theta}=f\left([X, Y]_{\theta^{\prime}}\right),(\forall) X, Y \in \Gamma\left(\theta^{\prime}\right)$.

Proposition 3.1. If $\left(\theta^{\prime}, D^{\prime},[\cdot, \cdot]_{\theta^{\prime}}\right)$ is an $A L S,\left(\theta, D,[\cdot, \cdot]_{\theta}\right)$ is a generalized algebroid and $f: \theta^{\prime} \rightarrow \theta$ is an anchored vector bundle morphism, then the map $f_{(\infty)}: \theta^{\prime(\infty)} \rightarrow \theta$ is a generalized algebroid morphism.

Proof. First, let $X, Y \in \Gamma\left(\theta^{\prime}\right)$. Then $\left[f_{(\infty)}(X), f_{(\infty)}(Y)\right]_{\theta^{(\infty)}}=\left[f_{0}(X), f_{0}(Y)\right]_{\theta}$ and $f_{(\infty)}\left([X, Y]_{\theta^{\prime}(\infty)}\right)=f_{(\infty)}\left([X, Y]_{\theta^{\prime}}+X \wedge_{1} Y\right)=f_{0}\left([X, Y]_{\theta^{\prime}}\right)+f_{1}\left(X \wedge_{1} Y\right)=f_{0}\left([X, Y]_{\theta^{\prime}}\right)+$ $\left[f_{0}(X), f_{0}(Y)\right]_{\theta}-f_{0}\left([X, Y]_{\theta^{\prime}}\right)=\left[f_{0}(X), f_{0}(Y)\right]_{\theta}$. In the same way, for $p \geq 0$, we have $\left[f_{(\infty)}\left(X_{p}\right), f_{(\infty)}\left(Y_{p}\right)\right]_{\theta}=f_{(\infty)}\left(\left[X_{p}, Y_{p}\right]_{\theta^{\prime}(\infty)}\right),(\forall) X_{p}, Y_{p} \in \Gamma\left(\theta^{\prime(p)}\right)$. The additivity of $f_{(\infty)}$ leads to the same relation for every $X, Y \in \Gamma\left(\theta^{\prime(\infty)}\right)$.

Proposition 3.2. Let $f: \theta^{\prime} \rightarrow \theta$ be an anchored vector bundle morphism of two $A L S$ 's over the same base. Then a generalized algebroid morphism $f_{(\infty)}: \theta^{\prime(\infty)} \rightarrow \theta^{(\infty)}$ is induced.

Proof. First $f$ can be extended to $f^{\prime}: \theta^{\prime} \rightarrow \theta^{(\infty)}, f^{\prime}=i \circ f$, where $i: \theta \rightarrow \theta^{(\infty)}$ is the inclusion morphism. Then using Proposition 3.1 one takes $f_{(\infty)}=f_{(\infty)}^{\prime}$.

Notice that Proposition 3.1 is not a particular case of the above Proposition because the brackets on $\theta$ and $\theta^{(\infty)}$ are not the same.

Proposition 3.3. If $i: \theta \rightarrow \theta$ is the identity morphism of an $A L S\left(\theta, D,[\cdot, \cdot]_{\theta}\right)$, then $i_{(\infty)}: \theta^{(\infty)} \rightarrow \theta^{(\infty)}$ is the identity map.

Proof. It suffices to show that if $X_{p}, Y_{p} \in \Gamma\left(\theta^{(p)}\right)$, where $p=\max \left(\operatorname{deg} X_{p}, \operatorname{deg} Y_{p}\right)$ then $i_{(\infty)}\left(X_{p} \wedge_{p+1} Y_{p}\right)=X_{p} \wedge_{p+1} Y_{p}$. Indeed, using induction and taking into account the 
definitions of $i_{(\infty)}$ and of the bracket on $\theta^{(p+1)}$ we have $i_{(\infty)}\left(X_{p} \wedge_{p+1} Y_{p}\right)=i_{p+1}\left(X_{p} \wedge_{p+1}\right.$ $\left.Y_{p}\right)=\left[X_{p}, Y_{p}\right]_{\theta^{(p+1)}}-\left[X_{p}, Y_{p}\right]_{\theta^{(p)}}=X_{p} \wedge_{p+1} Y_{p}$

Proposition 3.4. If $\left(\theta^{\prime \prime}, D^{\prime \prime},[\cdot, \cdot]_{\theta^{\prime \prime}}\right) \stackrel{f^{\prime}}{\rightarrow}\left(\theta^{\prime}, D^{\prime},[\cdot, \cdot]_{\theta^{\prime}}\right) \stackrel{f}{\rightarrow}\left(\theta, D,[\cdot, \cdot]_{\theta}\right)$ are morphisms of ALS's over the same manifold, then $\left(f^{\prime} \circ f\right)_{(\infty)}=f_{(\infty)}^{\prime} \circ f_{(\infty)}$.

Proof. The proof is essentially the same as that of Theorem 3.2.

Proposition 3.5. Let $[\cdot, \cdot]_{1}$ and $[\cdot, \cdot]_{2}$ be two brackets that correspond to the same anchor on the anchored vector bundle $(\theta, D)$. Then the generalized algebroids $\theta_{1}^{(\infty)}$ and $\theta_{2}^{(\infty)}$ which correspond to the ALS's are isomorphic.

Proof. The identity $i$ of $\theta$ is an anchored vector bundle morphism in four different ways. It extends to $i_{11(\infty)}=i d_{\theta_{1}^{(\infty)}}: \theta_{1}^{(\infty)} \rightarrow \theta_{1}^{(\infty)}, i_{22(\infty)}=i d_{\theta_{2}^{(\infty)}}: \theta_{2}^{(\infty)} \rightarrow \theta_{2}^{(\infty)}$, $i_{12(\infty)}: \theta_{1}^{(\infty)} \rightarrow \theta_{2}^{(\infty)}$ and $i_{21(\infty)}: \theta_{2}^{(\infty)} \rightarrow \theta_{1}^{(\infty)}$. Using the above results it follows that $i_{12(\infty)}$ and $i_{21(\infty)}$ are inverse to each other, where the indices are according to the brackets.

A similar argument can be used in order to prove the following result.

Proposition 3.6. If $\nabla$ and $\nabla^{\prime}$ are two linear $R$-connections on an $A V B(\theta, D)$, then the generalized algebroids which correspond to them are isomorphic.

We can summarize all these in the following result:

THEOREM 3.1. If $(\theta, D)$ is an anchored vector bundle, then $\theta^{(\infty)}$ belongs to an isomorphism class of generalized algebroids, which does not depend on the linear connections or the brackets.

The correspondences $(\theta, D) \rightarrow\left(\theta^{(\infty)}, D^{(\infty)},[\cdot, \cdot]_{\theta^{(\infty)}}\right)$ and $f \rightarrow f_{(\infty)}$ define a covariant functor from the category of anchored vector bundles to the category of generalized algebroids over the same base. It induces also a functor from the category of almost Lie structures to the category of generalized algebroids over the same base.

Notice that if $\left(\theta, D,[\cdot, \cdot]_{\theta}\right)$ is an algebroid, then it is a quotient algebroid of $\left(\theta^{(\infty)}, D^{(\infty)}\right.$, $\left.[\cdot, \cdot]_{\theta(\infty)}\right)$. The projection morphism is $i_{(\infty)}: \theta^{(\infty)} \rightarrow \theta$, where $i$ is the identity map of $\theta$. Notice also that the inclusion $j: \theta \rightarrow \theta^{(\infty)}$ is a right splitting of $i_{(\infty)}$, i.e. $i_{(\infty)} \circ j=i$, but it is never an algebroid morphism since $[j(X), j(Y)]_{\theta(\infty)}=[X, Y]_{\theta}+X \wedge_{1} Y$ and $j\left([X, Y]_{\theta}\right)=[X, Y]_{\theta},(\forall) X, Y \in \Gamma(\theta)$.

Now we extend the functor to both categories of anchored vector bundles (covariant and contravariant ones).

There are two categories which have as objects the vector bundles, but different morphisms. One of these categories of vector bundles is the usual one, when the morphisms are the usual morphisms of vector bundles $\xi^{\prime} \stackrel{(g, f)}{\rightarrow} \xi$ : if the vector bundles are $\xi^{\prime}=\left(E^{\prime}, \pi^{\prime}, M^{\prime}\right)$ and $\xi=(E, \pi, M), g: M^{\prime} \rightarrow M$ and $f: E^{\prime} \rightarrow E$ are such that $g \circ \pi^{\prime}=\pi \circ f$ and $f$ restricted to fibres, $f_{\mid \pi^{-1}\left(x^{\prime}\right)}: \pi^{\prime-1}\left(x^{\prime}\right) \rightarrow \pi^{-1}\left(g\left(x^{\prime}\right)\right)$, is linear. The other category of vector bundles has as morphisms the comorphisms of vector bundles $\xi^{\prime} \rightarrow(g, f) \xi$, $g: M^{\prime} \rightarrow M$ and $f: E \rightarrow E^{\prime}$ are such that $g \circ \pi^{\prime} \circ f=\pi$ and $f$ restricted to fibres, $f_{\mid \pi^{-1}\left(g\left(x^{\prime}\right)\right)}: \pi^{-1}\left(g\left(x^{\prime}\right)\right) \rightarrow \pi^{\prime-1}\left(x^{\prime}\right)$ is linear. 
Notice that for the vector bundles over the same base, where $g$ is the identity of the base, the two categories of vector bundles have the same morphisms.

One can also use the module morphisms of sections (see [4] for more details). If $\xi=$ $(E, \pi, M)$ is a vector bundle, then $(\mathcal{F}(M), \Gamma(\xi))$ is a module. A morphism of vector bundles $\xi^{\prime} \stackrel{(g, f)}{\rightarrow} \xi$ defines and it is defined by a con-morphism of modules $\left(\mathcal{F}\left(M^{\prime}\right), \Gamma\left(\xi^{\prime}\right)\right) \stackrel{\left(g^{*}, f_{*}\right)}{\rightarrow}$ $(\mathcal{F}(M), \Gamma(\xi))$, i.e. $g^{*}: \mathcal{F}(M) \rightarrow \mathcal{F}\left(M^{\prime}\right), g^{*}(u)=u \circ g$ and $f_{*}: \Gamma\left(\xi^{\prime}\right) \rightarrow \mathcal{F}\left(M^{\prime}\right) \otimes_{\mathcal{F}(M)} \Gamma(\xi)$, which is a morphism of $\mathcal{F}\left(M^{\prime}\right)$-modules. Notice that there is an isomorphism of modules $\mathcal{F}\left(M^{\prime}\right) \otimes_{\mathcal{F}(M)} \Gamma(\xi) \cong \Gamma\left(f^{*} \xi\right)$. A comorphism of vector bundles $\xi^{\prime} \underset{(g, f)}{\rightarrow} \xi$ defines and it is defined by a cov-morphism of modules $\left(\mathcal{F}\left(M^{\prime}\right), \Gamma\left(\xi^{\prime}\right)\right) \underset{\left(g^{*}, f_{*}\right)}{\rightarrow}(\mathcal{F}(M), \Gamma(\xi))$, i.e. $g^{*}: \mathcal{F}(M) \rightarrow \mathcal{F}\left(M^{\prime}\right), g^{*}(u)=u \circ f_{0}$ and $f^{*}: \Gamma(\xi) \rightarrow \Gamma\left(\xi^{\prime}\right)$, which is a morphism of $\mathcal{F}(M)$-modules.

All these can be adapted for morphisms of allowed vector bundles.

Let $\left(\theta^{\prime}, D^{\prime}\right)$ and $(\theta, D)$ be two anchored vector bundles over the bases $M^{\prime}$ and $M$ respectively. A comorphism of anchored vector bundles

$$
\left(\theta^{\prime}, D^{\prime}\right) \underset{(g, f)}{\rightarrow}(\theta, D)
$$

is a comorphism of vector bundles $\theta^{\prime} \underset{(g, f)}{\rightarrow} \theta$ (or a cov-morphism of modules $\left(\mathcal{F}\left(M^{\prime}\right), \Gamma\left(\theta^{\prime}\right)\right.$ ) $\left.\underset{\left(g^{*}, f^{*}\right)}{\rightarrow}(\mathcal{F}(M), \Gamma(\theta))\right)$ such that if $X \in \Gamma(\theta)$ and $u \in \mathcal{F}(M)$ then we have $D(X)(u)=$ $D^{\prime}\left(f^{*}(X)\right)\left(g^{*}(u)\right)$, or $g^{*}\left([X, u]_{\theta}\right)=\left[f^{*}(X), g^{*}(u)\right]_{\theta^{\prime}}$. A morphism of anchored vector bundles

$$
\left(\theta^{\prime}, D^{\prime}\right) \stackrel{(g, f)}{\longrightarrow}(\theta, D)
$$

is a morphism of vector bundles $\theta^{\prime} \stackrel{(g, f)}{\longrightarrow} \theta$ (or a con-morphism of modules $\left(\mathcal{F}\left(M^{\prime}\right), \Gamma\left(\theta^{\prime}\right)\right.$ ) $\left.\stackrel{\left(g, f_{*}\right)}{\longrightarrow}(\mathcal{F}(M), \Gamma(\theta))\right)$ such that if $u \in \mathcal{F}(M)$ and $X^{\prime} \in \Gamma\left(\theta^{\prime}\right)$ allows the decomposition

$$
f_{*}\left(X^{\prime}\right)=\sum_{i} a_{i}^{\prime} \otimes_{\mathcal{F}(M)} X_{i} \in \mathcal{F}\left(M^{\prime}\right) \otimes_{\mathcal{F}(M)} \Gamma(\theta),
$$

then $\left[X^{\prime}, g^{*}(u)\right]_{\theta^{\prime}}=\sum_{i} a_{i}^{\prime} \cdot g^{*}\left(\left[X_{i}, u\right]_{\theta}\right)$.

If $\left(\theta^{\prime}, D^{\prime},[\cdot, \cdot]_{\theta^{\prime}}\right)$ and $\left(\theta, D,[\cdot, \cdot]_{\theta}\right)$ are ALS's, then a comorphism of almost Lie structures is a comorphism of the anchored vector bundles, such that $(\forall) X, Y \in \Gamma(\theta)$ :

$$
\left[f^{*}(X), f^{*}(Y)\right]_{\theta^{\prime}}=f^{*}\left([X, Y]_{\theta}\right) .
$$

A morphism of almost Lie structures is a morphism of anchored vector bundles, such that $(\forall) X^{\prime}, Y^{\prime} \in \Gamma\left(\theta^{\prime}\right)$ which allow the decompositions (8) and

$$
f_{*}\left(Y^{\prime}\right)=\sum_{\alpha} a_{\alpha}^{\prime} \otimes_{\mathcal{F}(M)} Y_{\alpha} \in \mathcal{F}\left(M^{\prime}\right) \otimes_{\mathcal{F}(M)} \Gamma(\theta),
$$

respectively, then:

$$
\begin{aligned}
f_{*}\left(\left[X^{\prime}, Y^{\prime}\right]_{\theta^{\prime}}\right)= & \sum_{\alpha}\left[X^{\prime}, b_{\alpha}^{\prime}\right]_{\theta^{\prime}} \otimes_{\mathcal{F}(M)} Y_{\alpha}-\sum_{i}\left[Y^{\prime}, a_{i}^{\prime}\right]_{\theta^{\prime}} \otimes_{\mathcal{F}(M)} X_{i} \\
& +\sum_{i, \alpha} a_{i}^{\prime} b_{\alpha}^{\prime} \otimes_{\mathcal{F}(M)}\left[X_{i}, Y_{\alpha}\right] .
\end{aligned}
$$


Morphisms of algebroids and (Lie) algebroids are the very morphisms of almost Lie structures, forgetting the restrictive conditions for brackets. In the sequel we consider comorphisms and morphisms of vector bundles with differentials (i.e. anchored vector bundles, almost Lie structures, algebroids or Lie algebroids), simply called (co)morphisms.

Using the tensor representations, one can define in an analogous way morphisms of allowed vector bundles with differentials. However, in the sequel we need only morphisms of generalized algebroids and generalized Lie algebroids.

Consider an $\operatorname{ALS}\left(\theta^{\prime}, D^{\prime},[\cdot, \cdot]_{\theta^{\prime}}\right)$, an algebroid $\left(\theta, D,[\cdot, \cdot]_{\theta}\right)$ and $(g, f)$ a $($ co)morphism of ALS.

First we consider the case when $(g, f)$ is a comorphism.

Proposition 3.7. If $(g, f)$ is a comorphism of anchored vector bundles, where $\left(\theta^{\prime}, D^{\prime}\right.$, $\left.[\cdot, \cdot]_{\theta^{\prime}}\right)$ is an $A L S$ and $\left(\theta, D,[\cdot, \cdot]_{\theta}\right)$ is a generalized algebroid, then there is a generalized algebroid comorphism $\left(\theta^{\prime(\infty)}, D^{\prime(\infty)},[\cdot, \cdot]_{\theta^{\prime}(\infty)}\right) \underset{\left(g, f_{(\infty)}\right)}{\longrightarrow}\left(\theta, D,[\cdot, \cdot]_{\theta}\right)$.

Proof. A map $f_{(\infty)}: \theta^{\prime(\infty)} \rightarrow \theta$ can be constructed using the same ideas as in the case $M^{\prime}=M$ and $g=i d_{M}$ (see Proposition 3.1). Using the same arguments as in the proof of Proposition 3.1, a comorphism $\left(\theta^{\prime(\infty)}, D^{\prime(\infty)},[\cdot, \cdot]_{\theta^{\prime}(\infty)}\right) \underset{\left(g, f_{(\infty)}\right)}{\longrightarrow}\left(\theta, D,[\cdot, \cdot]_{\theta}\right)$ can be constructed.

Proposition 3.8. If $(g, f)$ is a comorphism of anchored vector bundles, where $\left(\theta^{\prime}, D^{\prime}\right.$, $\left.[\cdot, \cdot]_{\theta^{\prime}}\right)$ and $\left(\theta, D,[\cdot, \cdot]_{\theta}\right)$ are $A L S$ 's, then there is a generalized algebroid comorphism $\left(\theta^{\prime(\infty)}, D^{\prime(\infty)},[\cdot, \cdot]_{\theta^{\prime}(\infty)}\right) \underset{\left(g, f_{\infty}\right)}{\longrightarrow}\left(\theta^{(\infty)}, D^{(\infty)},[\cdot, \cdot]_{\theta^{(\infty)}}\right)$.

Proof. We have $\theta \subset \theta^{(\infty)}$, as an anchored vector subbundle; denote as $i: \theta \rightarrow \theta^{(\infty)}$ the inclusion, consider the anchored module morphism $\left(\theta^{\prime}, D^{\prime},[\cdot, \cdot]_{\theta^{\prime}}\right) \underset{(g, f \circ i)}{\rightarrow}\left(\theta^{(\infty)}, D^{(\infty)}\right.$, $\left.[\cdot, \cdot]_{\theta(\infty)}\right)$, then take $f_{(\infty)}=(f \circ i)_{(\infty)}$ using Proposition 3.7.

We deal now with morphisms. Let $\left(\theta^{\prime}, D^{\prime},[\cdot, \cdot]_{\theta^{\prime}}\right) \stackrel{(g, f)}{\rightarrow}\left(\theta, D,[\cdot, \cdot]_{\theta}\right)$ be a morphism of the $\operatorname{ALS}\left(\theta^{\prime}, D^{\prime},[\cdot, \cdot]_{\theta^{\prime}}\right)$ and an algebroid $\left(\theta, D,[\cdot, \cdot]_{\theta}\right)$. Denote $f=f_{0}$ and define $f_{1}: \theta^{\prime(1)} \rightarrow \mathcal{F}\left(M^{\prime}\right) \otimes_{\mathcal{F}(M)} \Gamma(\theta), f_{1 \mid \theta^{\prime}}=f_{0}$ and for $X^{\prime}, Y^{\prime} \in \Gamma\left(\theta^{\prime}\right)$ which allow the $f_{*}$-decompositions given by (8) and (9), then let $f_{1}\left(X^{\prime} \wedge Y^{\prime}\right)=\sum_{i} a_{i}^{\prime} b_{\alpha}^{\prime} \otimes\left[X_{i}, Y_{\alpha}\right]_{\theta}+$ $\sum_{\alpha}\left[X^{\prime}, b_{\alpha}^{\prime}\right]_{\theta^{\prime}} \otimes Y_{\alpha}-\sum_{i}\left[Y^{\prime}, a_{i}^{\prime}\right]_{\theta^{\prime}} \otimes X_{i}-f_{0}\left(\left[X^{\prime}, Y^{\prime}\right]_{\theta^{\prime}}\right)$. For every $a \in \mathcal{F}(M)$ we have:

$$
\begin{gathered}
{\left[X^{\prime} \wedge Y^{\prime}, g^{*}(a)\right]_{\theta^{\prime(1)}}=\left[D^{(0)}\left(X^{\prime}\right), D^{(0)}\left(Y^{\prime}\right)\right]\left(g^{*}(a)\right)-\left[\left[X^{\prime}, Y^{\prime}\right]_{\theta^{\prime}}, g^{*}(a)\right]_{\theta^{\prime}}=} \\
\left.\left[X^{\prime},\left[Y^{\prime}, g^{*}(a)\right]_{\theta^{\prime}}\right]_{\theta^{\prime}}-\left[Y^{\prime},\left[X^{\prime}, g^{*}(a)\right]_{\theta^{\prime}}\right]\right]_{\theta^{\prime}}-\left[\left[X^{\prime}, Y^{\prime}\right]_{\theta^{\prime}}, g^{*}(a)\right]_{\theta^{\prime}}= \\
\sum_{i} a_{i}^{\prime} b_{\alpha}^{\prime} g^{*}\left(\left[X_{i},\left[Y_{\alpha}, a\right]\right]-\left[Y_{\alpha},\left[X_{i}, a\right]\right]\right)+\sum_{\alpha}\left[X^{\prime}, b_{\alpha}^{\prime}\right]_{\theta^{\prime}} g^{*}\left(\left[Y_{\alpha}, a\right]\right)- \\
\sum_{i}\left[Y^{\prime}, a_{i}^{\prime}\right]_{\theta^{\prime}} g^{*}\left(\left[X_{i}, a\right]\right)-\left[\left[X^{\prime}, Y^{\prime}\right]_{\theta^{\prime}}, g^{*}(a)\right]_{\theta^{\prime}}=\sum_{i} a_{i}^{\prime} b_{\alpha}^{\prime} g^{*}\left(\left[\left[X_{i}, Y_{\alpha}\right], a\right]\right) \\
+\sum_{\alpha}\left[X^{\prime}, b_{\alpha}^{\prime}\right]_{\theta^{\prime}} g^{*}\left(\left[Y_{\alpha}, a\right]\right)-\sum_{i}\left[Y^{\prime}, a_{i}^{\prime}\right]_{\theta^{\prime}} g^{*}\left(\left[X_{i}, a\right]\right)-\left[\left[X^{\prime}, Y^{\prime}\right]_{\theta^{\prime}}, g^{*}(a)\right]_{\theta^{\prime}},
\end{gathered}
$$

$(\forall) X^{\prime}, Y^{\prime} \in \Gamma\left(\theta^{\prime}\right)$, thus $\left(\theta^{\prime(1)}, D^{(1)}\right) \stackrel{\left(g, f_{1}\right)}{\longrightarrow}(\theta, D)$ is an anchored vector bundle morphism. 
Inductively, assume that $f_{k}$ is constructed, then let $f_{k+1}: \theta^{\prime(k+1)} \rightarrow \theta, f_{k+1 \mid \theta^{\prime(k)}}=f_{k}$ and $f_{k+1}\left(X^{\prime} \wedge Y^{\prime}\right)=\sum_{i} a_{i}^{\prime} b_{\alpha}^{\prime} \otimes\left[X_{i}, Y_{\alpha}\right]_{\theta}+\sum_{\alpha}\left[X^{\prime}, b_{\alpha}^{\prime}\right]_{\theta^{\prime}} \otimes Y_{\alpha}-\sum_{i}\left[Y^{\prime}, a_{i}^{\prime}\right]_{\theta^{\prime}} \otimes X_{i}-$ $f_{k}\left(\left[X^{\prime}, Y^{\prime}\right]_{\theta^{\prime}}\right),(\forall) X^{\prime}, Y^{\prime} \in \Gamma\left(\theta^{\prime(k)}\right)$, where $X^{\prime}$ and $Y^{\prime}$ have $\left(f:=f_{k}\right)$-decompositions (8) and (9). In the same way as in the case $k=1$ it follows that $f_{k}$ is an anchored vector bundle morphism. The maps $\left(f_{k}\right)_{k \in \mathbb{N}}$ define a map $f_{(\infty)}: \theta^{\prime(\infty)} \rightarrow g^{*} \theta$.

Proposition 3.9. If $\left(\theta, D,[\cdot, \cdot]_{\theta}\right)$ is a (generalized) algebroid and $\left(\theta^{\prime}, D^{\prime}\right) \stackrel{(g, f)}{\longrightarrow}(\theta, D)$ is a morphism of anchored vector bundles, then $\left(\theta^{\prime(\infty)}, D^{\prime(\infty)},[\cdot, \cdot]_{\theta^{\prime}(\infty)}\right) \stackrel{\left(g, f_{(\infty)}\right)}{\longrightarrow}\left(\theta, D,[\cdot, \cdot]_{\theta}\right)$ is a generalized algebroid morphism.

Proof. First, let $X^{\prime}, Y^{\prime} \in \Gamma\left(\theta^{\prime}\right)$ which have $f_{*}$-decompositions (8) and (9) respectively. Then $f_{(\infty)}\left(\left[X^{\prime}, Y^{\prime}\right]_{\theta^{\prime}(\infty)}\right)=f_{1}\left(\left[X^{\prime}, Y^{\prime}\right]_{\theta^{\prime}(1)}\right)=f_{1}\left(\left[X^{\prime}, Y^{\prime}\right]_{\theta^{\prime}}+X^{\prime} \wedge_{1} Y^{\prime}\right)=\sum_{i} a_{i}^{\prime} b_{\alpha}^{\prime} \otimes$ $\left[X_{i}, Y_{\alpha}\right]_{\theta}+\sum_{\alpha}\left[X^{\prime}, b_{\alpha}^{\prime}\right]_{\theta^{\prime}} \otimes Y_{\alpha}-\sum_{i}\left[Y^{\prime}, a_{i}^{\prime}\right]_{\theta^{\prime}} \otimes X_{i}$. In the same way, if $k \in \mathbb{N}, p \in \mathbb{N}^{*}$ and $X^{\prime}, Y^{\prime} \in \theta^{\prime(k)}$, where $k=\max \left(\operatorname{deg} X^{\prime}, \operatorname{deg} Y^{\prime}\right)$ have $\left(f:=f_{k}\right)$-decompositions (8) and (9) respectively, then

$$
\begin{aligned}
f_{(\infty)}\left(\left[X^{\prime}, Y^{\prime}\right]_{\theta^{\prime}(\infty)}\right) & =f_{k+1}\left(\left[X^{\prime}, Y^{\prime}\right]_{\theta^{\prime}(k)}+X^{\prime} \wedge_{k} Y^{\prime}\right) \\
& =f_{k}\left(\left[X^{\prime}, Y^{\prime}\right]_{\theta^{\prime}(k)}\right)+f_{k+1}\left(X^{\prime} \wedge_{k} Y^{\prime}\right) \\
& =\sum_{i} a_{i}^{\prime} b_{\alpha}^{\prime} \otimes\left[X_{i}, Y_{\alpha}\right]_{\theta}+\sum_{\alpha}\left[X^{\prime}, b_{\alpha}^{\prime}\right]_{\theta^{\prime}(k)} \otimes Y_{\alpha}-\sum_{i}\left[Y^{\prime}, a_{i}^{\prime}\right]_{\theta^{\prime}(k)} \otimes X .
\end{aligned}
$$

The additivity of $f_{(\infty)}$ leads to the same relation for every $X^{\prime}, Y^{\prime} \in \Gamma\left(\theta^{\prime(\infty)}\right)$ which have $\left(f:=f_{(\infty)}\right)$-decompositions (8) and (9) respectively.

Notice that the above result extends Proposition 3.2.

Proposition 3.10. If $\left(\theta^{\prime}, D^{\prime},[\cdot, \cdot]_{\theta^{\prime}}\right) \stackrel{(g, f)}{\longrightarrow}\left(\theta, D,[\cdot, \cdot]_{\theta}\right)$ is an anchored vector bundle morphism, then a generalized algebroid morphism

$$
\left(\theta^{\prime(\infty)}, D^{\prime(\infty)},[\cdot, \cdot]_{\theta^{\prime}(\infty)}\right) \stackrel{\left(g, f_{(\infty)}\right)}{\longrightarrow}\left(\theta^{(\infty)}, D^{(\infty)},[\cdot, \cdot]_{\theta^{(\infty)}}\right)
$$

is induced. If $\left(\theta^{\prime}, D^{\prime},[\cdot, \cdot]_{\theta^{\prime}}\right) \underset{(g, f)}{\longrightarrow}\left(\theta, D,[\cdot, \cdot]_{\theta}\right)$ is an anchored vector bundle comorphism, then a generalized algebroid comorphism

$$
\left(\theta^{\prime(\infty)}, D^{\prime(\infty)},[\cdot, \cdot]_{\theta^{\prime}(\infty)}\right) \underset{\left(g, f_{(\infty)}\right)}{\longrightarrow}\left(\theta^{(\infty)}, D^{(\infty)},[\cdot, \cdot]_{\theta^{(\infty)}}\right)
$$

is induced.

Proof. For morphisms one uses Proposition 3.9 and for comorphisms one use Proposition 3.7. In both cases one considers the inclusion $i: \theta \rightarrow \theta^{(\infty)}$ which is a morphism of anchored vector bundles.

THEOREM 3.2. The associations $(\theta, D) \rightarrow\left(\theta^{(\infty)}, D^{(\infty)},[\cdot, \cdot]_{\theta^{(\infty)}}\right)$ and $(g, f) \mapsto\left(g, f_{(\infty)}\right)$ define two functors from the two categories of anchored vector bundle in the corresponding categories of generalized algebroids.

Proof. In the case of comorphisms the proof is similar to the case of the vector bundles over the same base, essentially as the proof below. In the case of morphisms we have to 
show that if $\left(\theta^{\prime \prime}, D^{\prime \prime}\right) \stackrel{\left(g^{\prime}, f^{\prime}\right)}{\longrightarrow}\left(\theta^{\prime}, D^{\prime}\right) \stackrel{(g, f)}{\longrightarrow}(\theta, D)$ are anchored vector bundle morphisms, then $\left(g, f_{(\infty)}\right) \circ\left(g^{\prime}, f_{(\infty)}^{\prime}\right)=\left(g \circ g^{\prime},\left(f \circ f^{\prime}\right)_{(\infty)}\right)$. We use the tensor representations of sections.

Consider $X^{\prime \prime}, Y^{\prime \prime} \in \Gamma\left(\theta^{\prime \prime}\right)$ which allow the $f^{\prime}\left(=f_{(\infty)}^{\prime}\right)$-decompositions $f^{\prime}\left(X^{\prime \prime}\right)=$ $f_{(\infty)}^{\prime}\left(X^{\prime \prime}\right)=\sum_{i} a_{i}^{\prime \prime} \otimes X_{i}^{\prime}$ and $f^{\prime}\left(Y^{\prime \prime}\right)=f_{(\infty)}^{\prime}\left(Y^{\prime}\right)=\sum_{\alpha} b_{\alpha}^{\prime \prime} \otimes Y_{\alpha}^{\prime}$ respectively. Then $f_{(\infty)}^{\prime}\left(X^{\prime \prime} \wedge_{1} Y^{\prime \prime}\right)=f_{1}^{\prime}\left(X^{\prime \prime} \wedge_{1} Y^{\prime \prime}\right)=\sum_{i, \alpha} a_{i}^{\prime \prime} b_{\alpha}^{\prime \prime} \otimes\left[X_{i}^{\prime}, Y_{\alpha}^{\prime}\right]_{\theta^{\prime}(\infty)}+\sum_{\alpha}\left[X^{\prime \prime}, b_{\alpha}^{\prime \prime}\right]_{\theta^{\prime \prime}} \otimes Y_{\alpha}^{\prime}-$ $\sum_{i}\left[Y^{\prime \prime}, a_{i}^{\prime \prime}\right]_{\theta^{\prime \prime}} \otimes X_{i}^{\prime}-f_{0}^{\prime}\left(\left[X^{\prime \prime}, Y^{\prime \prime}\right]_{\theta^{\prime \prime}(\infty)}\right)$. Since $\left(g, f_{(\infty)}\right)$ is a generalized algebroid morphism, it follows that if $X_{i}^{\prime}$ and $Y_{\alpha}^{\prime}$ have the $f\left(=f_{(\infty)}\right)$-decompositions $f\left(X_{i}^{\prime}\right)=f_{(\infty)}\left(X_{i}^{\prime}\right)=$ $\sum_{u} c_{i u}^{\prime} \otimes Z_{i u}$ and $f\left(Y_{\alpha}^{\prime}\right)=f_{(\infty)}\left(Y_{\alpha}^{\prime}\right)=\sum_{v} d_{\alpha v}^{\prime} \otimes T_{\alpha v}$ respectively, then $\left.f_{(\infty)}\left(\left[X_{i}^{\prime}, Y_{\alpha}^{\prime}\right]_{\theta^{\prime}(\infty)}\right]\right)$ $=\sum_{u, v} c_{i u}^{\prime} d_{\alpha v}^{\prime} \otimes\left[Z_{i u}, T_{\alpha v}\right]_{\theta(\infty)}+\sum_{v}\left[X_{i}^{\prime}, d_{\alpha v}^{\prime}\right] \otimes T_{\alpha v}-\sum_{u}\left[Y_{\alpha}^{\prime}, c_{i u}^{\prime}\right] \otimes Z_{i u}$. It follows that

$$
\begin{aligned}
f_{(\infty)} \circ f_{(\infty)}^{\prime}\left(X^{\prime \prime} \wedge_{1} Y^{\prime \prime}\right)= & \sum_{i, \alpha, u, v} a_{i}^{\prime \prime} b_{\alpha}^{\prime \prime} \varphi^{\prime}\left(c_{i u}\right) \varphi^{\prime}\left(d_{\alpha v}\right) \otimes\left[Z_{i u}, T_{\alpha v}\right]_{\theta(\infty)} \\
& +\sum_{i, \alpha, v} a_{i}^{\prime \prime} b_{\alpha}^{\prime \prime} \varphi^{\prime}\left(\left[X_{i}^{\prime}, d_{\alpha v}^{\prime}\right]_{\theta^{\prime}}\right) \otimes T_{\alpha v} \\
& -\sum_{i, \alpha, u} a_{i}^{\prime \prime} b_{\alpha}^{\prime \prime} \varphi^{\prime}\left(\left[Y_{\alpha}^{\prime}, c_{i u}^{\prime}\right]_{\theta^{\prime}}\right) \otimes Z_{i u} \\
& +\sum_{i, \alpha, v}\left[X^{\prime \prime}, b_{\alpha}^{\prime \prime}\right]_{\theta^{\prime \prime}} \varphi^{\prime}\left(d_{\alpha v}^{\prime}\right) \otimes T_{\alpha v} \\
& -\sum_{i}\left[Y^{\prime \prime}, a_{i}^{\prime \prime}\right]_{\theta^{\prime \prime}} \varphi^{\prime}\left(c_{i u}^{\prime}\right) \otimes Z_{i u}-f \circ f_{0}^{\prime}\left(\left[X^{\prime \prime}, Y^{\prime \prime}\right]_{\theta^{\prime \prime}}\right) \\
= & \sum_{i, \alpha, u, v} a_{i}^{\prime \prime} \varphi^{\prime}\left(c_{i u}\right) b_{\alpha}^{\prime \prime} \varphi^{\prime}\left(d_{\alpha v}\right) \otimes\left[Z_{i u}, T_{\alpha v}\right]_{\theta}(\infty) \\
& +\sum_{\alpha, v}\left[X^{\prime \prime}, b_{\alpha}^{\prime \prime} \cdot \varphi^{\prime}\left(d_{\alpha v}^{\prime}\right)\right]_{\theta^{\prime \prime}} \otimes T_{\alpha v} \\
& -\sum_{i, u}\left[Y^{\prime \prime}, a_{i}^{\prime \prime} \cdot \varphi^{\prime}\left(c_{i u}^{\prime}\right)\right]_{\theta^{\prime \prime}} \otimes Z_{i u}-f_{0} \circ f_{0}^{\prime}\left(\left[X^{\prime \prime}, Y^{\prime \prime}\right]_{\theta^{\prime \prime}}\right) \\
= & \left(f \circ f^{\prime}\right)(\infty)\left(X^{\prime \prime} \wedge_{1} Y^{\prime \prime}\right) .
\end{aligned}
$$

An analogous calculation can be performed in higher degrees.

Summarizing, we have the following result, which extends Theorem 3.1:

THEOREM 3.3. If $(\theta, D)$ is an anchored vector bundle, then $\theta^{(\infty)}$ belongs to an isomorphism class of generalized algebroids, which does not depend on the linear connections or the brackets.

The correspondences $(\theta, D) \rightarrow\left(\theta^{(\infty)}, D^{(\infty)},[\cdot, \cdot]_{\theta^{(\infty)}}\right)$ and $(g, f) \rightarrow\left(g, f_{(\infty)}\right)$ define two covariant functors from the two categories of anchored vector bundles in the corresponding two categories of generalized algebroids. They induce also functors from the two categories of almost Lie structures in the corresponding categories of generalized algebroids.

A particular case is when the anchor on $\theta$ is null. In this case one has an algebroid structure given by a skew-symmetric map $b: \theta \wedge \theta \rightarrow \theta$. The generalized algebroid $\theta^{(\infty)}$ does not depend on $b$. In particular the map $b$ can be chosen null. 
4. The derived Lie algebroid of an anchored vector bundle. A special case for an anchored vector bundle is when the vector bundle is parallelisable, for example, when the vector bundle is restricted to a domain of a vectorial chart. In this case, the bracket and the adapted linear R-connection can be taken null when restricted to the local base. According to Theorems 3.1 and 3.3, the derived algebroid constructed using this particular bracket is isomorphic to any other derived algebroid using another bracket or another linear R-connection. Consider the $\mathcal{F}(M)$-submodule $\mathcal{M}_{\mathcal{J}} \subset \Gamma\left(\theta^{(\infty)}\right)$ generated by the Jacobiator $\mathcal{J}(\theta)=\left\{\mathcal{J}(X, Y, Z) \mid X, Y, Z \in \Gamma\left(\theta^{(\infty)}\right)\right\}$ and successive brackets of elements from $\Gamma\left(\theta^{(\infty)}\right)$, but starting from $\mathcal{J}(\theta)$. Taking into account the construction of the derived algebroid, this module does not depend on the anchor; there is also a generalized subalgebroid $\left(\theta_{\mathcal{L}}, D_{0}\right) \subset\left(\theta^{(\infty)}, \theta^{(\infty)}\right)$, which has a null anchor $\left(D_{0}\right.$ is $\theta^{(\infty)}$ restricted to $\left.\theta_{\mathcal{L}}\right)$, such that $\Gamma\left(\theta_{\mathcal{L}}\right)$ is just $\mathcal{M}_{\mathcal{J}}$. We can consider the quotient generalized vector bundle $\theta^{\infty}=\theta^{(\infty)} / \theta_{\mathcal{L}}$; since the anchor $D_{0}$ is null and the bracket $[\cdot, \cdot]_{\theta(\infty)}$ has the property that $[X, Y]_{\theta} \in \mathcal{M}_{\mathcal{L}}=\Gamma\left(\theta_{\mathcal{L}}\right)$ whenever $X \in \Gamma\left(\theta_{\mathcal{L}}\right)$ and $Y \in \Gamma\left(\theta^{(\infty)}\right)$, it follows that $D^{(\infty)}$ induces an anchor $D^{\infty}$ and the bracket $[\cdot, \cdot]_{\theta(\infty)}$ induces a bracket $[\cdot, \cdot]_{\theta^{\infty}}$ on $\theta^{\infty}$, which becomes a Lie algebroid. This local construction can be extended for any anchored vector bundle $(\theta, D)$. We call $\left(\theta^{\infty}, D^{\infty}(\theta),[\cdot, \cdot]_{\theta}\right)$ the derived Lie algebroid of $(\theta, D)$.

The following result is an analogue of Theorem 3.3:

THEOREM 4.1. If $(\theta, D)$ is an anchored vector bundle, then $\theta^{\infty}$ belongs to an isomorphism class of generalized Lie algebroids, which does not depend on the linear connections or the brackets.

The correspondences $(\theta, D) \rightarrow\left(\theta^{\infty}, D^{\infty},[\cdot, \cdot]_{\theta^{\infty}}\right)$ and $(g, f) \rightarrow\left(g, f_{\infty}\right)$ define two covariant functors from the two categories of anchored vector bundles in the corresponding two categories of generalized Lie algebroids. The correspondences above induce also functors from the two categories of almost Lie structures in the corresponding categories of generalized Lie algebroids.

If $\left(\theta^{\prime}, D^{\prime}\right)$ is an anchored vector subbundle of $(\theta, D)$, then it is natural to know if there is a similar relation between the derived (Lie) algebroids.

THEOREM 4.2. If $\left(\theta^{\prime}, D^{\prime}\right)$ is an anchored vector subbundle of $(\theta, D)$, then $\left(\theta^{\prime(\infty)}, D^{\prime(\infty)}\right.$, $\left.[\cdot, \cdot]_{\theta^{\prime}(\infty)}\right)$ is a subalgebroid of $\left(\theta^{(\infty)}, D^{(\infty)}(\theta),[\cdot, \cdot]_{\theta^{(\infty)}}\right)$ and $\left(\theta^{\prime \infty}, D^{\prime \infty},[\cdot, \cdot]_{\theta^{\prime \infty}}\right)$ is a Lie subalgebroid of $\left(\theta^{\infty}, D^{\infty}(\theta),[\cdot, \cdot]_{\theta^{\infty}}\right)$.

Proof. Let $\theta^{\prime \prime} \subset \theta$ be a vector subbundle such that $\theta$ can be reduced as $\theta^{\prime} \oplus \theta^{\prime \prime}$, $\Pi^{\prime}, \Pi^{\prime \prime}: \theta \rightarrow \theta$ be the natural projectors on $\theta^{\prime}$ and $\theta^{\prime \prime}$ respectively and $\bar{\nabla}$ be a linear R-connection on $\theta$. If $X \in \Gamma(\theta)$, denote as $X=X^{\prime}+X^{\prime \prime}$ the decomposition according to the reduction $\theta=\theta^{\prime} \oplus \theta^{\prime \prime}$ (i.e. $X^{\prime}=\Pi^{\prime}(X)$ and $X^{\prime \prime}=\Pi^{\prime \prime}(X)$ ). The linear R-connections $\nabla$ and $\nabla^{\prime}$ on $\theta$ and $\theta^{\prime}$ respectively, defined by:

$$
\begin{aligned}
\nabla_{X^{\prime}+X^{\prime \prime}}\left(Y^{\prime}+Y^{\prime \prime}\right) & =\Pi^{\prime}\left(\nabla_{X^{\prime}} Y^{\prime}\right)+\nabla_{X^{\prime}} Y^{\prime \prime}+\nabla_{X^{\prime \prime}} Y^{\prime}+\nabla_{X^{\prime \prime}} Y^{\prime \prime}, \\
\nabla_{X^{\prime}}^{\prime} Y^{\prime} & =\Pi^{\prime}\left(\nabla_{X^{\prime}} Y^{\prime}\right),
\end{aligned}
$$

fulfill the condition

$$
\nabla_{X^{\prime}} Y^{\prime}=\nabla_{X^{\prime}}^{\prime} Y^{\prime},(\forall) X^{\prime}, Y^{\prime} \in \Gamma\left(\theta^{\prime}\right)
$$


Consider now the brackets $[\cdot, \cdot]_{\theta}$ and $[\cdot, \cdot]_{\theta^{\prime}}$ such that the linear R-connections $\nabla$ and $\nabla^{\prime}$ respectively are torsion free. Taking account of the constructions of the derived generalized (Lie) algebroids the conclusion follows.

Using Propositions 3.9 and 3.7, the following universal properties of the derived Lie algebroid can be proved.

THEOREM 4.3. If $\left(\theta, D,[\cdot, \cdot]_{\theta}\right)$ is a (generalized Lie) algebroid and $\left(\theta^{\prime}, D^{\prime}\right) \stackrel{(g, f)}{\longrightarrow}(\theta, D)$ is a morphism of anchored vector bundles, then $\left(\theta^{\prime \infty}, D^{\prime \infty},[\cdot, \cdot]_{\theta^{\prime}(\infty)}\right) \stackrel{\left(g, f_{\infty}\right)}{\longrightarrow}\left(\theta, D,[\cdot, \cdot]_{\theta}\right)$ is the unique generalized Lie algebroid morphism such that $(g, f)=\left(g, f_{\infty}\right) \circ\left(i d_{M}, i\right)$, where $\left(\theta^{\prime}, D^{\prime},[\cdot, \cdot]_{\theta^{\prime}}\right) \stackrel{\left(i d_{M}, i\right)}{\longrightarrow}\left(\theta^{\prime \infty}, D^{\prime \infty},[\cdot, \cdot]_{\theta^{\prime}(\infty)}\right)$ is the canonical inclusion morphism of anchored vector bundles.

5. Anchored vector bundles and foliations. It is well-known that the distribution defined by an algebroid integrates to a Stefan-Sussmann foliation (see for example [1] or $[15])$. The following example shows that the distribution defined by a generalized Lie algebroid fails to integrate to a Stefan-Sussmann foliation. Consider the smooth function

$$
\varphi(t)=\left\{\begin{array}{cc}
e^{-1 / t^{2}} & \text { if } t>0, \\
0 & \text { if } t \leq 0,
\end{array}\right.
$$

and define an anchor $D: \theta=\tau \mathbb{R}^{2} \rightarrow \tau \mathbb{R}^{2}$ by

$$
D\left(u(x, y) \frac{\partial}{\partial x}+v(x, y) \frac{\partial}{\partial y}\right)=u(x, y) \frac{\partial}{\partial x}+\varphi(x) \cdot v(x, y) \frac{\partial}{\partial y} .
$$

The distribution defined by this anchor is the horizontal line for $x \leq 0$ and the whole $\mathbb{R}^{2}$ for $x>0$. It is obvious that it is not integrable in the sense of Stefan-Sussmann. It is easily to check that the $\mathcal{F}\left(\mathbb{R}^{2}\right)$-submodule $M^{p}=D^{\{p\}}\left(\Gamma\left(\theta^{(p)}\right)\right) \subset \mathcal{X}\left(\mathbb{R}^{2}\right)$ is generated by the vector fields

$$
\left\{\frac{\partial}{\partial x}, \varphi(x) \frac{\partial}{\partial y}, \varphi^{\prime}(x) \frac{\partial}{\partial y}, \varphi^{\prime \prime}(x) \frac{\partial}{\partial y}, \ldots, \varphi^{(p)}(x) \frac{\partial}{\partial y}\right\}
$$

which are $\mathcal{F}\left(\mathbb{R}^{2}\right)$-linearly independent in $M^{p}$. We have

$$
M^{0} \subsetneq M^{1} \subsetneq \cdots \subsetneq M^{p} \subsetneq \cdots \subsetneq M^{\infty}=D^{(\infty)}\left(\Gamma\left(\theta^{(\infty)}\right)\right)
$$

but they are equal as distributions. Obviously the resulting distribution is not integrable and $D^{(\infty)}\left(\Gamma\left(\theta^{(\infty)}\right)\right)$ is not finitely generated.

Another example can be considered for the function

$$
\varphi(t)=\left\{\begin{array}{cc}
e^{-1 / t^{2}} & \text { if } t \neq 0 \\
0 & \text { if } t=0
\end{array}\right.
$$

The anchor $D: \theta=\tau \mathbb{R}^{2} \rightarrow \tau \mathbb{R}^{2}$ can be also considered using formula (10). Then the distribution defined by this anchor is integrable, but the module of vector fields $D(\Gamma(\theta))$ is not involutive; the involutive module it generates is $D^{(\infty)}\left(\Gamma\left(\theta^{(\infty)}\right)\right)$, which is not finitely generated.

These two examples show that the construction of $\theta^{(\infty)}$ is motivated at least by the reason to include the two kinds of distributions in those studied by (Lie) algebroids. 
The sequence (11) can be considered also for every anchored vector bundle $(\theta, D)$. We say that this sequence is stationary if there is $n \in \mathbb{N}$ such that $M^{n}=M^{n+1}=\cdots=M^{\infty}$.

THEOREM 5.1. If for an anchored vector bundle $(\theta, D)$ the sequence (11) is stationary, then the distribution defined by $D^{(\infty)}\left(\Gamma\left(\theta^{(\infty)}\right)\right)=D^{\infty}\left(\Gamma\left(\theta^{\infty}\right)\right)$ is integrable in the sense of Stefan-Sussmann.

Proof. The hypothesis implies that there is $n \in \mathbb{N}$ such that $D^{(\infty)}\left(\Gamma\left(\theta^{(\infty)}\right)\right)=$ $D^{(n)}\left(\Gamma\left(\theta^{(n)}\right)\right)$, thus $D^{(\infty)}\left(\Gamma\left(\theta^{(\infty)}\right)\right)$ is finitely generated. The proof of the integrability uses similar ideas as for Lie algebroids in [1] or as for generalized algebroids in [15].

6. Lifts of linear connections and differential forms. In this $\operatorname{section}\left(\theta, D,[\cdot, \cdot]_{\theta}\right)$ is an almost Lie structure.

Proposition 6.1. Let $\theta^{\prime}$ be a vector bundle and $\nabla: \Gamma(\theta) \times \Gamma\left(\theta^{\prime}\right) \rightarrow \Gamma\left(\theta^{\prime}\right)$ be a linear $R$-connection on $\theta$. Then there is a linear $R$-connection $\nabla^{(\infty)}: \Gamma\left(\theta^{(\infty)}\right) \times \Gamma\left(\theta^{\prime}\right) \rightarrow \Gamma\left(\theta^{\prime}\right)$ which is curvature free and extends $\nabla$.

Proof. Consider the derived vector bundles $\theta^{(n)}, n \geq 0$. Denote $\nabla=\nabla^{(0)}$ and $\theta=\theta^{(0)}$. First we define the linear connection $\nabla^{(1)}: \Gamma\left(\theta^{(1)}\right) \times \Gamma\left(\theta^{\prime}\right) \rightarrow \Gamma\left(\theta^{\prime}\right), \nabla_{\mid \theta^{(0)}}^{(1)}=\nabla^{(0)}$ and $\nabla_{X \wedge_{1} Y}^{(1)} X^{\prime}=\nabla_{X}^{(0)} \nabla_{Y}^{(0)} X^{\prime}-\nabla_{Y}^{(0)} \nabla_{X}^{(0)} X^{\prime}-\nabla_{[X, Y]_{\theta}(0)}^{(0)} X^{\prime},(\forall) X, Y \in \Gamma\left(\theta^{(0)}\right), X^{\prime} \in \Gamma\left(\theta^{\prime}\right)$, then inductively we construct in the same way, for every $k \in \mathbb{N}$, the linear connections $\nabla^{(k)}$ : $\Gamma\left(\theta^{(k)}\right) \times \Gamma\left(\theta^{\prime}\right) \rightarrow \Gamma\left(\theta^{\prime}\right)$. These linear connections define $\nabla^{(\infty)}: \Gamma\left(\theta^{(\infty)}\right) \times \Gamma\left(\theta^{\prime}\right) \rightarrow \Gamma\left(\theta^{\prime}\right)$ as follows: if $X^{\prime} \in \Gamma\left(\theta^{\prime}\right)$ and $X \in \Gamma\left(\theta^{(p)}\right)$ have a degree $p \geq 0$, then $\nabla_{X}^{(\infty)} X^{\prime}=\nabla_{X}^{(p)} X^{\prime}$.

It follows that if $k$ is the greatest degree of $X$ and $Y$, then $\nabla_{X}^{(\infty)} \nabla_{Y}^{(\infty)} X^{\prime}-\nabla_{Y}^{(\infty)} \nabla_{X}^{(\infty)} X^{\prime}$ $-\nabla_{[X, Y]_{\theta}(\infty)}^{(\infty)} X^{\prime}=\nabla_{X}^{(k)} \nabla_{Y}^{(k)} X^{\prime}-\nabla_{Y}^{(k)} \nabla_{X}^{(k)} X^{\prime}-\nabla_{[X, Y]_{\theta}(\infty)}^{(\infty)} X^{\prime}=\nabla_{X}^{(k)} \nabla_{Y}^{(k)} X^{\prime}-\nabla_{Y}^{(k)} \nabla_{X}^{(k)} X^{\prime}-$ $\nabla_{[X, Y]_{\theta}(k)}^{(\infty)}+X \wedge_{k+1} X^{\prime}=\nabla_{X}^{(k)} \nabla_{Y}^{(k)} X^{\prime}-\nabla_{Y}^{(k)} \nabla_{X}^{(k)} X^{\prime}-\nabla_{[X, Y]_{\theta}(k)}^{(k+2)}+X \wedge_{k+1} Y X^{\prime}=0$.

We say that the linear connection $\nabla^{(\infty)}$ is the lift of $\nabla$ to $\theta^{(\infty)}$.

Proposition 6.2. Let $\theta^{\prime}$ be a vector bundle and $\nabla: \Gamma(\theta) \times \Gamma\left(\theta^{\prime}\right) \rightarrow \Gamma\left(\theta^{\prime}\right)$ be a linear $R$-connection on $\theta$. Then there is a linear $R$-connection $\nabla^{\infty}: \Gamma\left(\theta^{\infty}\right) \times \Gamma\left(\theta^{\prime}\right) \rightarrow \Gamma\left(\theta^{\prime}\right)$ which is curvature free and extends $\nabla$.

Proof. It suffices to prove that

$$
\nabla_{X}^{(\infty)} X^{\prime}=0,(\forall) X \in \mathcal{M}_{\mathcal{J}}, X^{\prime} \in \Gamma\left(\theta^{\prime}\right) .
$$

Then $\nabla^{\infty}$ can be defined using $\nabla^{(\infty)}$ in an obvious manner as $\nabla_{\Pi(Y)}^{\infty} X^{\prime}=\nabla_{\Pi Y}^{(\infty)} X^{\prime}$, where $\Pi: \Gamma\left(\theta^{(\infty)}\right) \rightarrow \Gamma\left(\theta^{\infty}\right)$ is the canonical projection.

In order to prove the assertion (12), consider first $X \in \mathcal{J}(\theta)=\{\mathcal{J}(A, B, C) \mid A, B, C \in$ $\left.\Gamma\left(\theta^{(\infty)}\right)\right\}$. Then using that $\nabla^{(\infty)}$ is curvature free, it follows easily that $\nabla_{\mathcal{J}(A, B, C)}^{(\infty)} X^{\prime}=0$. Consider now $X=[A, B]_{\theta^{(\infty)}}$, where $A \in \mathcal{M}_{\mathcal{J}}$ has the property $\nabla_{A}^{(\infty)} X^{\prime}=0$ for any $X^{\prime} \in \Gamma\left(\theta^{\prime}\right)$ and $B \in \Gamma\left(\theta^{(\infty)}\right)$; then $\nabla_{[A, B]}^{(\infty)} X^{\prime}=\nabla_{A}^{(\infty)} \nabla_{B}^{(\infty)} X^{\prime}-\nabla_{B}^{(\infty)} \nabla_{A}^{(\infty)} X^{\prime}=0$. The assertion (12) follows.

Consider now an allowed vector bundle $\theta$ over $M$. For $k \geq 0$, let $\wedge^{k} \theta^{*}$ be the exterior vector bundle of order $k$ of $\theta^{*}$. A section of $\Gamma\left(\wedge^{k} \theta^{*}\right)$ can be regarded as a skew symmetric 
and $\mathcal{F}(M)$-linear map $\omega: \Gamma(\theta)^{k} \rightarrow \mathcal{F}(M)$. Let us suppose that on $\theta$ are given an anchor $D$ and a bracket $[\cdot, \cdot]_{\theta}$ which corresponds to $D$. Define

$$
\begin{gathered}
d_{\theta}: \wedge^{k} \theta^{*} \longrightarrow \wedge^{k+1} \theta^{*}: \\
\left(d_{\theta} \omega\right)\left(X_{0}, \ldots, X_{k}\right)=\sum_{i}(-1)^{i}\left[X_{i}, \omega\left(X_{1}, \ldots, \hat{X}_{i}, \ldots, X_{k}\right)\right]_{\theta} \\
+\sum_{i<j}(-1)^{i+j} \omega\left(\left[X_{i}, X_{j}\right]_{\theta}, X_{0}, \ldots, \hat{X}_{i}, \ldots, \hat{X}_{j}, \ldots, X_{k}\right) .
\end{gathered}
$$

It is well-defined and satisfies $d_{\theta}^{2}=0 \mathrm{iff}\left(\theta, D,[\cdot, \cdot]_{\theta}\right)$ is a generalized Lie algebroid.

Let $\left(\theta, D,[\cdot, \cdot]_{\theta}\right)$ be a generalized algebroid. Then $d_{\theta}^{2} f=0$ only for $f \in \mathcal{F}(M)$. Consider the ideal $I \subset \Gamma\left(\wedge^{*} \theta^{*}\right)$ generated by the image of $d_{\theta}^{2}$. Then $d$ can be extended to a 1-degree derivation $\tilde{d}$ on $\tilde{\Gamma}^{*}=\Gamma\left(\wedge^{*} \theta^{*}\right) / I$, so $\left(\tilde{\Gamma}^{*}, \tilde{d}\right)$ is a graded differential complex. The cohomology of this differential complex can be called the algebroid cohomology of $L$, and we denote it by $H^{\bullet}(\theta)$.

Consider now a generalized algebroid $\left(\theta, D,[\cdot, \cdot]_{\theta}\right)$, an allowed vector bundle $\xi$ over the same base $M$ as $\theta$ and a linear connection $\nabla$ on $\xi$ related to $\theta$. We suppose that $\nabla$ is curvature free. We can define

$$
\tilde{\Gamma}^{n}\left(\theta^{*}, \xi\right)=\tilde{\Gamma}^{n} \otimes \xi .
$$

The linear connection $\nabla$ can be regarded as defined by the $\mathcal{F}(M)$-linear map:

$$
\delta: \tilde{\Gamma}^{0}\left(\theta^{*}, \xi\right)=\Gamma(\xi) \longrightarrow \tilde{\Gamma}^{1}\left(\theta^{*}, \xi\right), \delta(m)(X)=\nabla_{X} m .
$$

Using $\tilde{d}$, we can extend $\delta$ to a map

$$
\delta: \quad \tilde{\Gamma}^{n}\left(\theta^{*}, \xi\right) \rightarrow \tilde{\Gamma}^{n+1}\left(\theta^{*}, \xi\right)
$$

by the rule

$$
\delta(\omega \otimes s)=\tilde{d} \omega \otimes s+(-1)^{n} \omega \otimes \delta s,
$$

where $\omega \in \tilde{\Gamma}^{n}\left(\theta^{*}\right)$ and $s \in \Gamma(\xi)$. Then $\delta^{2}=0$ iff $(\theta, D, L)$ is a generalized Lie algebroid; the cohomology of $\left(\tilde{\Gamma}^{*}\left(\theta^{*}, \xi\right), \delta\right)$ is called in this case the cohomology of $\theta$ with coefficients in $\xi$.

The operator $\delta$ satisfies

$$
\delta((\omega \wedge \eta) \otimes s)=(\tilde{d} \omega \wedge \eta) \otimes s+(-1)^{|\omega|} \omega \wedge \delta(\eta \otimes s)
$$

for $\omega, \eta \in \tilde{\Gamma}^{*} L^{*}$ and $s \in \Gamma(\xi)$.

Consider now an (allowed) vector bundle $\xi$ and a linear connection $\nabla$ on $\xi$ related to an $\operatorname{ALS}\left(\theta, D,[\cdot, \cdot]_{\theta}\right)$. According to Proposition 6.1 , the linear connection $\nabla$ lifts to a linear connection $\nabla^{(\infty)}$ on $\xi$, related to the Lie pseudoalgebra $\theta^{(\infty)}$, which is curvature free. It follows that we can consider the differential complex of $\theta^{(\infty)}$ with coefficients in $\xi$, related to $\nabla$.

A special case is when $\xi$ is the trivial bundle $\left(M \times \mathbb{R}, p r_{1}, M\right)$, when $\Gamma(\xi)=\mathcal{F}(M)$, $\nabla_{X} a=[X, f]_{L},(\forall) X \in \Gamma(\theta)$ and $a \in \mathcal{F}(M)$. Then the curvature of $\nabla$ is $\nabla_{X \wedge Y} a=$ $\mathcal{D}(X \wedge Y)(a)$; it is null only if $\left(\theta, D,[\cdot, \cdot]_{\theta}\right)$ is a generalized algebroid. The lifted connection $\nabla^{(\infty)}$ is $\nabla_{X_{(\infty)}}^{(\infty)} a=D^{(\infty)}\left(X_{(\infty)}\right)(a),(\forall) X_{(\infty)} \in \Gamma\left(\theta^{(\infty)}\right)$ and $a \in \mathcal{F}(M)$. It follows that 
the differential complex of $\theta^{(\infty)}$ with coefficients in $\left(M \times \mathbb{R}, p r_{1}, M\right)$, related to this connection, is just the differential complex of $\theta^{(\infty)}$ with real cofficients.

Let $\omega \in \Gamma\left(\theta^{*}\right)=\Gamma\left(\wedge^{1} \theta^{*}\right)$ be a 1-form. Denote $\omega=\omega_{(0)}$ and define $\omega_{(1)} \in \Gamma\left(\Lambda^{1}\left(\theta^{(1)}\right)\right)$ by $\omega_{(1)}(X)=\omega_{(0)}(X), \omega_{(1)}\left(X \wedge_{1} Y\right)=d_{\theta^{(0)}} \omega_{(0)}(X, Y),(\forall) X, Y \in \Gamma(\theta)=\Gamma\left(\theta^{(0)}\right)$. Inductively, assume that $\omega_{(k)}$ is defined for $k \geq 1$, then define $\omega_{(k+1)} \in \Gamma\left(\Lambda^{1}\left(\theta^{(k+1)}\right)\right)$ by $\omega_{(k+1)}(X)=\omega_{(k)}(X), \omega_{(k+1)}\left(X \wedge_{1} Y\right)=d_{\theta^{(k)}} \omega_{(k)}(X, Y),(\forall) X, Y \in \Gamma\left(\theta^{(k)}\right)$. These forms define $\omega_{(\infty)} \in \Gamma\left(\Lambda^{1}\left(\theta^{(\infty)}\right)\right)$ which define to its turn $\omega_{(\infty)} \in \Gamma\left(\Lambda^{1}\left(\theta^{(\infty)}\right)\right)$ by restriction.

Let us show that the form $\omega_{(\infty)}$ is closed, i.e. $d_{(\infty)} \omega_{(\infty)}=0$. Indeed, if $X, Y \in$ $\Gamma\left(\theta^{(k)}\right)$ then $\omega_{(k+2)}\left(X \wedge_{k+2} Y\right)=\left[X, \omega_{(k+1)}(Y)\right]-\left[Y, \omega_{(k+1)}(X)\right]-\omega_{(k+1)}\left([X, Y]_{\theta^{(k+1)}}\right)=$ $\left[X, \omega_{(k)}(Y)\right]-\left[Y, \omega_{(k)}(X)\right]-\omega_{(k+1)}\left([X, Y]_{\theta^{(k)}}+X \wedge_{k+1} Y\right)=0$; it follows that if $X, Y \in$ $\Gamma\left(\theta^{(k)}\right)$ then $d_{(\infty)} \omega_{(\infty)}(X, Y)=\left[X, \omega_{(\infty)}(Y)\right]_{(\infty)}-\left[Y, \omega_{(\infty)}(X)\right]_{(\infty)}-\omega_{(\infty)}\left([X, Y]_{(\infty)}\right)=$ $\left[X, \omega_{(k)}(Y)\right]_{(k)}-\left[Y, \omega_{(k)}(X)\right]_{(k)}-\omega_{(\infty)}\left([X, Y]_{(k)}+X \wedge_{k+1} Y\right)=0$, thus $d_{(\infty)} \omega_{(\infty)}=0$.

This construction can be extended to any $p$-form.

Proposition 6.3. For $p \geq 1$ any $p$-form $\omega \in \Gamma\left(\wedge^{p} \theta^{*}\right)$ can be extended canonically to a closed $p$-form $\omega_{(\infty)} \in \Gamma\left(\wedge^{p} \theta^{(\infty) *}\right)$.

Proof. Denote $\omega=\omega_{(0)}$ and define $\omega_{(1)} \in \Gamma\left(\Lambda^{p}\left(\theta^{(1)}\right)\right)$ by

$\omega_{(1)}\left(X_{1}, \ldots, X_{p}\right)=\omega_{(0)}\left(X_{1}, \ldots, X_{p}\right)$,

$\omega_{(1)}\left(X_{0} \wedge_{1} X_{1}, X_{2}, \ldots, X_{p}\right)=-\frac{2}{p(p-1)} d_{\theta^{(0)}} \omega_{(0)}\left(X_{0}, X_{1}, X_{2}, \ldots, X_{p}\right)$,

$(\forall) X_{0}, \ldots, X_{p} \in \Gamma(\theta)=\Gamma\left(\theta^{(0)}\right)$ and 0 in the other cases. Inductively, assume that $\omega_{(k)}$ is defined for $k \geq 1$, then define $\omega_{(k+1)} \in \Gamma\left(\Lambda^{p}\left(\theta^{(k+1)}\right)\right)$ by

$\omega_{(k+1)}\left(X_{1}, \ldots, X_{p}\right)=\omega_{(k)}\left(X_{1}, \ldots, X_{p}\right)$,

$\omega_{(k+1)}\left(X_{0} \wedge_{1} X_{1}, X_{2}, \ldots, X_{p}\right)=-\frac{1}{N} d_{\theta^{(k)}} \omega_{(k)}\left(X_{0}, X_{1}, X_{2}, \ldots, X_{p}\right),(\forall) X_{0}, \ldots, X_{p} \in$ $\Gamma\left(\theta^{(k)}\right)$, all homogeneous (i.e. $X_{i} \in \Gamma\left(\theta^{(s)}\right) \backslash \Gamma\left(\theta^{(s-1)}\right)$ and we say that $X_{i}$ has degree $\left.s\right)$ and 0 otherwise, where $N$ is the number of the couples $\left(X_{i}, X_{j}\right), i \neq j$, such that $X_{i}$ or $X_{j}$ has degree $k$. These forms define $\omega_{(\infty)} \in \Gamma\left(\Lambda^{p}\left(\theta^{(\infty)}\right)\right)$.

If $X_{0}, \ldots, X_{p} \in \Gamma\left(\theta^{(k)}\right)$ then

$$
\begin{aligned}
& d_{(\infty)} \omega_{(\infty)}\left(X_{0}, \ldots,\right.\left.X_{p}\right)=d_{\theta(k)} \omega_{(k)}\left(X_{0}, X_{1}, X_{2}, \ldots, X_{p}\right) \\
&+\frac{1}{N} \sum_{i<j}(-1)^{i+j} \omega_{(k+1)}\left(X_{i} \wedge_{k+1} X_{j}, X_{0}, \ldots, \hat{X}_{i}, \ldots, \hat{X}_{j}, \ldots, X_{p}\right)=0 .
\end{aligned}
$$

We say that the $p$-form $\omega_{(\infty)}$ is the lift of the $p$-form $\omega$.

Every $p$-form $\omega \in \Gamma\left(\wedge^{p} \theta^{*}\right)$ define the $p$-form $\tilde{\omega}^{\prime} \in \Gamma\left(\wedge^{p} \theta^{(\infty) *}\right), \tilde{\omega}^{\prime}=\pi^{*} \omega$, where $\pi: \theta^{(\infty)} \rightarrow \theta$ is the canonical projection as vector bundle morphism. It is easy to see that $\tilde{\omega}=\omega_{(\infty)}$ iff $d \omega=0$. Consistent examples can be obtained in the case of the lift of a form $\omega \in \Lambda^{\text {top }}\left(\theta^{*}\right)$, when the lift is $\tilde{\omega}$ since $d_{\theta} \omega=0$.

The following result shows that concerning the lift the case $p=1$ is quite different from the case $p>1$.

Proposition 6.4. Any exact 1-form $\omega \in \wedge^{1} \Gamma\left(\theta^{(\infty) *}\right)$ is the lift of an exact 1-form. More precisely $\omega_{(\infty)}=d_{(\infty)} f$ iff $\omega=d_{\theta} f$.

Proof. For $k=0$ the equality $\omega_{(\infty)}=d_{(\infty)} f$ means that $\omega=d_{\theta} f$. Conversely, if $\omega=$ $d_{\theta} f$ then using induction it follows that $d_{\theta^{(k)}} \omega_{(k)}(X, Y)=\left[X \wedge_{k} Y, f\right]_{\theta^{(k)}}=\omega_{(k+1)}\left(X \wedge_{k+1}\right.$ 
$Y)$ for $k \geq 1$ and $X, Y \in \theta^{(k)}$, thus $\omega_{(\infty)}=d_{(\infty)} f$ and $\omega_{(\infty)}=d_{(\infty)} f$.

Proposition 6.5. For any closed 1-form $\omega \in \wedge^{1} \Gamma\left(\theta^{(\infty) *}\right)$ there is a closed 1-form $\omega^{\prime} \in \wedge^{1} \Gamma\left(\theta^{\infty *}\right)$ such that $\omega=\Pi^{*} \omega^{\prime}$, where $\Pi: \theta^{(\infty)} \rightarrow \theta^{\infty}$ is the canonical projection.

Proof. It suffices to prove that if $X \in \mathcal{M}_{\mathcal{J}}$ then $\omega(X)=0$. Since $\omega$ is closed, it follows that

$$
\omega\left([X, Y]_{\theta(\infty)}\right)=[X, \omega(Y)]_{\theta(\infty)}-[Y, \omega(X)]_{\theta(\infty)},
$$

$(\forall) X, Y \in \Gamma\left(\theta^{(\infty)}\right)$. Let us take $X, Y, Z \in \Gamma\left(\theta^{(\infty)}\right)$. Using this formula and the fact that $\left(\theta^{(\infty)}, D^{(\infty)}(\theta),[\cdot, \cdot]_{\theta(\infty)}\right)$ is an algebroid, it follows that

$$
\begin{aligned}
\omega\left(\left[[X, Y]_{\theta^{(\infty)}}, Z\right]_{\left.\theta^{(\infty)}\right)}\right)=\left[X,[Y, \omega(Z)]_{\theta(\infty)}\right]_{\theta(\infty)}-\left[Y,[X, \omega(Z)]_{\theta^{(\infty)}}\right]_{\theta^{(\infty)}} \\
\\
-\left[Z,[X, \omega(Y)]_{\theta^{(\infty)}}\right]_{\theta^{(\infty)}}+\left[Z,[Y, \omega(X)]_{\theta^{(\infty)}}\right]_{\theta^{(\infty)}} .
\end{aligned}
$$

Writing analogous formulas for $\omega\left(\left[[Y, Z]_{\theta^{(\infty)}}, X\right]_{\theta^{(\infty)}}\right)$ and $\omega\left(\left[[Z, X]_{\theta^{(\infty)}}, Y\right]_{\theta^{(\infty)}}\right)$, then summing, we obtain that $\omega\left(\mathcal{J}_{\theta(\infty)}(X, Y, Z)\right)=0$. Take now $X \in \Gamma\left(\theta^{(\infty)}\right)$ and $Y \in \mathcal{M}_{\mathcal{J}}$ such that $\omega(Y)=0$. Then using the formula (15) and using also that $D^{(\infty)}(Y)=0$, it follows that $\omega\left([X, Y]_{\theta(\infty)}\right)=0$. Thus $\omega(X)=0,(\forall) X \in \mathcal{M}_{\mathcal{J}}$.

Notice that analogous results for a $p$-form $\omega \in \wedge^{p} \theta^{(\infty) *}, p \geq 2$, seem to fail.

Notice also that an interesting application is in the case of the de Rham complex of a manifold $M$. Every antisymmetric form lifts to a closed form on $\tau M^{(\infty)}$. Every 1-form lifts also to a closed 1-form on $\tau M^{\infty}$, but not every $p$-form on $M(p \geq 2)$ lifts to a closed $p$-form on $\tau M^{\infty}$. We expect that the study of the differential complexes associated with $\tau M^{(\infty)}$ and $\tau M^{\infty}$ will give in the future more information about the manifold.

We give now an application, constructing the modular class of an ALS in the same way as in [5] for Lie algebroids. Consider an $\operatorname{ALS}(\theta, D,[\cdot, \cdot])$ and let $\xi$ be a line bundle over the same base $M$ as $\theta$. Then $\xi^{\prime}=\xi \otimes \xi$ is a trivial line bundle, thus $\Gamma\left(\xi^{\prime}\right)$ is 1-dimensional as an $\mathcal{F}(M)$-module. In this case let $s_{0} \in \Gamma\left(\xi^{\prime}\right)$ be such that $\Gamma\left(\xi^{\prime}\right)=\left\{f \cdot s_{0} \mid f \in \mathcal{F}(M)\right\}$. If $\nabla$ is a connection on $\xi$ related to $\theta$, then the formula:

$$
\nabla_{X} s_{0}=\omega(X) \cdot s_{0}, X \in \Gamma\left(\xi^{\prime}\right)
$$

defines $\omega \in \Lambda^{1}\left(\theta^{*}\right)$. Consider another element $s_{0}^{\prime}=f_{0} \cdot s_{0} \in \Gamma\left(\xi^{\prime}\right)$ which generates $\Gamma\left(\xi^{\prime}\right)$ and defines $\omega^{\prime} \in \Lambda^{1}\left(\left(\Gamma\left(\theta^{(\infty)}\right)^{*}\right)\right.$. Then $\omega^{\prime}=d_{\theta}\left(\left|f_{0}\right|+\omega\right.$. The connection $\nabla$ is curvature free iff $(\theta, D,[\cdot, \cdot])$ is an algebroid $(\mathcal{D}=0)$ and $d_{\theta} \omega=0$. Consider now the lifted connection $\nabla^{\infty}$ on $\xi^{\prime}$, related to $\theta^{(\infty)}$. An analogous formula (16) defines $\omega_{\infty} \in \Lambda^{1}\left(\left(\Gamma\left(\theta^{(\infty)}\right)\right)^{*}\right)$. Since $\nabla^{\infty}$ is curvature free, it follows that $\omega_{\infty}$ has $d_{\theta_{\infty}} \omega_{\infty}=0$, thus it is closed and defines a cohomology class $\left[\omega_{\infty}\right] \in H^{1}\left(\theta^{\infty}\right)$. We define the characteristic class of $\xi^{\prime}$ related to $\nabla$ as $\left[\omega_{\infty}\right]$, denoted as $\Theta_{\xi^{\prime}}$. If $\nabla$ is a linear R-connection on $\xi$, then it defines a linear R-connection $\nabla^{\prime}$ on $\xi^{\prime}=\xi \otimes \xi$. We define the characteristic class of $\xi$ related to $\nabla$ as $\frac{1}{2} \Theta_{\xi^{\prime}}$, where the characteristic class considered on $\xi^{\prime}$ is related to $\nabla^{\prime}$. As in [5], it remains to define a line bundle $\xi$ and a linear R-connection $\nabla$ on $\xi$ which is intrinsic. We can take several situations.

First, following [5] we take $\xi=\left(\wedge^{t o p} \theta\right) \otimes\left(\wedge^{t o p} \tau^{*} M\right)$ and for $\omega \otimes \omega^{\prime} \in \Gamma(\xi)$ and $X \in \Gamma(\theta)$ we define

$$
\nabla_{X}\left(\omega \otimes \omega^{\prime}\right)=L_{X} \omega \otimes \omega^{\prime}-\omega \otimes L_{D(X)} \omega^{\prime}
$$


where $D$ is the anchor of $\theta$ and $L_{X}, L_{D(X)}$ are the Lie derivatives on $\wedge^{*} \theta$ and $\wedge^{*} \tau^{*} M$ respectively. Other intrinsic definitions are $\xi^{\prime}=\left(\wedge^{t o p} \theta^{*}\right) \otimes\left(\wedge^{t o p} \tau M\right), \xi^{\prime \prime}=\left(\wedge^{t o p} \theta\right) \otimes$ $\left(\wedge^{t o p} \tau M\right)$ and $\xi^{\prime \prime \prime}=\left(\wedge^{t o p} \theta^{*}\right) \otimes\left(\wedge^{t o p} \tau^{*} M\right)$. The formula (17) defines also linear $\mathrm{R}$ connections on each of these vector bundles.

Acknowledgments. The authors thank the organizers of the Conference, especially Professor Jan Kubarski, for the invitation and their kind support.

\section{References}

[1] C. Albert and P. Dazord, Théorie des groupoïdes symplectiques, Publ. Dept. Math. Lyon I 4(1988), 51-105.

[2] A. Cannas da Silva and A. Weinstein, Lectures on Geometric Models for Noncommutative Algebras, University of California at Berkeley, December 1, 1998.

[3] C. M. de Barros, Opérateurs infinitésimaux sur l'algèbre des formes différentielles extérieures, C. R. Acad. Sci. Paris 261, 1965, 4594-4597.

[4] W. Greub, S. Halperin and R. Vanstone, Connections, Curvature and Cohomology, Vol. I, Academic Press, New York, 1972.

[5] S. Evens, J.-H. Lu and A. Weinstein, Transverse measures, the modular class, and a cohomology pairing for Lie algebroids, Quart. J. Math Oxford (2)50 (1999), 117-136, dg-ga 9610008.

[6] R. L. Fernandes, Lie algebroids, holonomy and characteristic classes, math-DG 0007132.

[7] P. J. Higgins and K. Mackenzie, Algebraic construction in the category of Lie algebroids, J. Algebra 129 (1990), 194-230.

[8] J. Kubarski, Bott vanishing theorem for regular Lie algebroids, Trans. Amer. Math. Soc. 348 (1996), 2151-2167.

[9] K. Mackenzie, Lie Groupoids and Lie Algebroids in Differential Geometry, London Mathematical Society Lecture Note Series 124, Cambridge Univ. Press, 1987.

[10] K. Mackenzie, Lie algebroids and Lie pseudoalgebras, Bull. London Math. Soc. 27 (1995), 97-147.

[11] R. Montgomery, M. Shapiro and A. Stolin, Chaotic geodesics in Carnot groups, dg-ga 9704013.

[12] P. Popescu, On the geometry of relative tangent spaces, Rev. Roum. Math. Pures Appl. 37 (1992), 727-733.

[13] P. Popescu, Almost Lie structures, derivations and R-curvature on relative tangent spaces, Rev. Roum. Math. Pures Appl. 37 (1992), 779-789.

[14] P. Popescu, Categories of modules with differentials, J. Algebra 185 (1996), 50-73.

[15] P. Popescu, On generalized algebroids, in: New Developments in Differential Geometry, Ed. J. Szenthe, Kluwer Academic Publ., 1998, 329-342.

[16] P. Popescu and M. Popescu, Embedding almost Lie structures, in: Proceedings of Summer School in Differential Geometry, September 3-7, 1999, Coimbra (Portugal), CIM Publications 2000, 149-157.

[17] P. Popescu, On anchored modules which allow linear connections (to appear).

[18] J. Pradines, Théorie de Lie pour les groupoïdes différentiables. Calcul différentiel dans la catégorie des groupoïdes infinitésimaux, C. R. Acad. Sci. Paris, Sér. A Math. 264 (1967), $245-248$. 
[19] M. Puta, Hamiltonian Mechanical Systems and Geometric Quantization, Kluwer Acad. Publ., 1993.

[20] D. Roytenberg and A. Weinstein, Courant algebroids and strongly homotopy Lie algebras, Lett. Math. Phys. 46 (1998), 81-93; math-QA/9802118.

[21] P. Stefan, Accessible sets, orbits and foliations with singularities, Proc. London Math. Soc. 29 (1974), 699-713.

[22] H. Y. Sussmann, Orbits of families of vector fields and integrability of distributions, Trans. Amer. Math. Soc. 180 (1973), 171-188.

[23] R. Swan, Topological examples of projective modules, Trans. Amer. Math. Soc. 250 (1977), 201-234.

[24] Gh. Vrănceanu, Sur quelques tenseurs dans les variétés non holonomes, C. R. Acad. Sci. Paris 186 (1929), 995.

[25] R. Wolak, The structure tensor of a transverse G-structure on a foliated manifold, Bolletino U.M.I. (7) 4-A(1990), 1-15. 